\title{
Strategies for the Generation of Molecularly Imprinted Polymeric Nitroxide Catalysts
}

Christopher D. Anderson, Kenneth J. Shea* and Scott D. Rychnovsky*

Department of Chemistry, 516 Rowland Hall, University of California, Irvine, CA 92697-2025

srychnov@uci.edu
A. General Experimental Details
$\mathrm{S} 2$
B. Template Synthesis
S3-S15
C. Polymerization
S16-S17
D. Oxidation Chemistry
$\mathrm{S} 18$
E. Representative EPR Spectra
S19
F. NMR Spectra
S20-S52 
A. General Experimental Details: IR spectra were recorded on a MIDAC Prospect FT-IR spectrometer. ${ }^{1} \mathrm{H}$ NMR were recorded at 500 and $400 \mathrm{MHz}$ and ${ }^{13} \mathrm{C}$ spectra were recorded at 125 and $100 \mathrm{MHz}$ on Bruker instruments. ${ }^{1} \mathrm{H}$ NMR and ${ }^{13} \mathrm{C}$ chemical shifts are reported as $\delta$ values in ppm relative to TMS. ${ }^{1} \mathrm{H}$ NMR coupling constants are reported in hertz and refer to apparent multiplicities and not true coupling constants. Muliplicity is indicated as follows: $s$ (singlet), $d$ (doublet), t (triplet), q (quartet), m (multiplet), dd (doublet of doublets), etc. EPR spectra were obtained using Bruker instruments at ambient temperature. Optical rotations were determined on a JASCO DIP-370 digital polarimeter; concentration $c$ is reported as $\mathrm{g} / 100 \mathrm{~mL}$. Combustion analyses were performed by M-H-W laboratories, Phoenix, AZ. Mass spectra were determined on an Fisions autospec spectrometer or a Micromass LCT electrospray mass spectrometer. Tetrahydrofuran (THF), $\mathrm{Et}_{2} \mathrm{O}$ and $\mathrm{CH}_{2} \mathrm{Cl}_{2}$ were dried by filtration through alumina according to the procedure described by Grubbs. ${ }^{1}$ Pyridine and triethyl amine were distilled from $\mathrm{KOH}$. Liquid chromatography was performed using forced flow (flash chromatography) of the indicated solvent system on Sorbent Technologies silica gel (230-450 mesh). ${ }^{2}$ Moisture sensitive reactions were carried out under an atmosphere of argon using oven or flame dried glassware and standard syringe/septa techniques.

\footnotetext{
${ }^{1}$ Pangborn, A. B.; Giardello, M. A.; Grubbs, R. H.; Rosen, R. K.; Timmers, F. J. Organometallics 1996, 15, 1518-1520.

${ }^{2}$ Still, W. C.; Kahn, M.; Mitra, A. J. Org. Chem. 1978, 43, 2923-2925.
} 


\section{B. Template Synthesis}

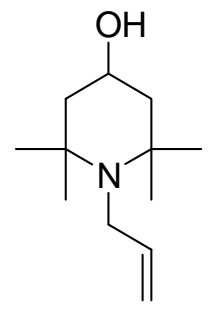

4-Hydroxy-1-(2-propenyl)-2,2,6,6-tetramethylpiperidine (S1). To a mixture of 4-hydroxy2,2,6,6-tetramethylpiperidine $(5.0 \mathrm{~g}, 39 \mathrm{mmol})$ and toluene $(13 \mathrm{~mL})$ in a heavy-walled tube was added allyl bromide $(1.59 \mathrm{~mL}, 19.6 \mathrm{mmol})$. The tube was sealed and placed in a $130{ }^{\circ} \mathrm{C}$ oil bath for $46 \mathrm{~h}$. The resultant mixture was cooled to ambient temperature, diluted with hexanes $(10 \mathrm{~mL})$ and was purified by flash chromatography $\left(\mathrm{SiO}_{2}, 20: 80\right.$ ethyl acetate:hexanes) to yield $2.8 \mathrm{~g}$ (73\% yield) of $\mathbf{S 1}$ as a white solid. m.p. $89-90^{\circ} \mathrm{C}$; $1 \mathrm{H}$ NMR (500 MHz, CDCl3) 5.79-5.88 (m, 1 H), 5.12-5.16 (m, 1 H), 4.91-4.95 (m, $1 \mathrm{H}), 3.94-4.00(\mathrm{~m}, 1 \mathrm{H}), 3.13-3.14(\mathrm{~m}, 2 \mathrm{H}), 1.82$ (dd, $J$ $=4.1,12.1,2 \mathrm{H}), 1.36(\mathrm{t}, J=11.5,2 \mathrm{H}), 1.08(\mathrm{~s}, 6 \mathrm{H}), 1.04(\mathrm{~s}, 6 \mathrm{H}) ;{ }^{13} \mathrm{C} \mathrm{NMR}(125 \mathrm{MHz}$, $\mathrm{CDCl}_{3}$ ) 143,3, 113.2, 64.5, 56.6, 50.6, 46.4, 34.4, 22.7; IR (film) 3290 (br), 2932, 1458, 1036 $\mathrm{cm}^{-1}$; HRMS (CI/NH 3$)$ Calcd for $\mathrm{C}_{12} \mathrm{H}_{23} \mathrm{NO}\left(\mathrm{M}^{+}\right)$197.1775, Found: 197.1780; Anal. Calcd for $\mathrm{C}_{12} \mathrm{H}_{23} \mathrm{NO}$ : C, 73.04; H, 11.75; N, 7.10. Found: C, 73.20; H, 11.76; N, 7.29.

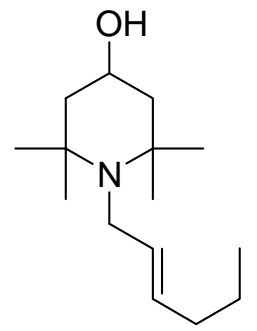

4-Hydroxy-1-(E-2-hexenyl)-2,2,6,6-tetramethylpiperidine (7). To a mixture of 4-hydroxy2,2,6,6-tetramethylpiperidine $(3.5 \mathrm{~g}, 22 \mathrm{mmol})$ and toluene $(5 \mathrm{~mL})$ in a heavy-walled tube was added E-1-bromo-2-hexene $(1.8 \mathrm{~g}, 11 \mathrm{mmol})$. The tube was sealed and the reaction mixture heated at $150{ }^{\circ} \mathrm{C}$ for $45 \mathrm{~h}$. The black reaction mixture was then cooled to ambient temperature and purified by flash chromatography $\left(\mathrm{SiO}_{2}, 20: 80\right.$ ethyl acetate:hexanes $\rightarrow 30: 70$ ethyl 
acetate:hexanes) to yield $1.7 \mathrm{~g}$ (53\% yield) of 7 as a light yellow oil. ${ }^{1} \mathrm{H}$ NMR (500 MHz, $\left.\mathrm{CDCl}_{3}\right) 5.36-5.48(\mathrm{~m}, 2 \mathrm{H}), 3.95$ (tdt, $\left.J=4.2,5.5,11.5,1 \mathrm{H}\right), 3.07$ (d, $\left.J=4.5,1 \mathrm{H}\right), 1.94(\mathrm{dt}, J=$ 6.4, 6.8, 2 H), 1.77-1.82 (m, 2 H), 1.31-1.41 (m, 5 H), 1.08 (s, 6 H), $1.02(\mathrm{~s}, 6 \mathrm{H}), 0.86$ (t, $J=$ 7.4, $3 \mathrm{H}) ;{ }^{13} \mathrm{C} \mathrm{NMR}\left(125 \mathrm{MHz}, \mathrm{CDCl}_{3}\right)$ 134.4, 128.8, 64.0, 56.1, 50.1, 45.3, 34.4, 34.1, 22.6, 22.2, 13.7; IR (film) 3337 (br), 2963, $1462 \mathrm{~cm}^{-1}$; HRMS (CI/ $\left.\mathrm{NH}_{3}\right)$ calcd for $\mathrm{C}_{15} \mathrm{H}_{29} \mathrm{NO}\left(\mathrm{M}^{+}\right)$ 239.2249, found: 239.2251 .

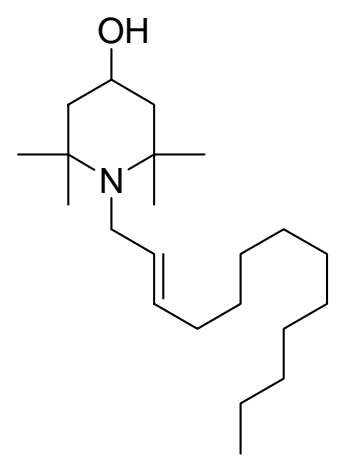

4-Hydroxy-1-(E-2-tridecenyl)-2,2,6,6-tetramethylpiperidine (S2). To a solution of E-1bromo-2-tridecene (4.2 g, $16 \mathrm{mmol})$ in toluene $(125 \mathrm{~mL})$ was added 4-hydroxy-2,2,6,6tetramethylpiperidine $(5.5 \mathrm{~g}, 35 \mathrm{mmol})$. The mixture was heated at reflux for $8 \mathrm{~d}$. The reaction mixture was cooled to ambient temperature, diluted with saturated aqueous $\mathrm{NaHCO}_{3}(50 \mathrm{~mL})$ and then extracted with ethyl acetate $(3 \times 25 \mathrm{~mL})$. The combined organics were dried over $\mathrm{Na}_{2} \mathrm{SO}_{4}$ and concentrated under reduced pressure. The residue was purified by flash chromatography $\left(\mathrm{SiO}_{2}, 15: 85\right.$ ethyl acetate:hexanes) to yield $2.8 \mathrm{~g}(51 \%) \mathrm{S} 2$ as an off-white solid. mp 45-47 ${ }^{\circ} \mathrm{C} ;{ }^{1} \mathrm{H}$ NMR (500 MHz, $\mathrm{CDCl}_{3}$ ) 5.34-5.46 (m, $2 \mathrm{H}$ ), 3.89-3.97 (m, $\left.1 \mathrm{H}\right), 3.05$ (d, $J=4.7,2$ H), 1.91-1.97 (m, 2 H), 1.78 (dd, $J=4.0,12.1,2$ H), 1.20-1.37 (m, 19 H), 1.07 (s, $6 \mathrm{H}), 1.01(\mathrm{~s}, 6 \mathrm{H}), 0.86(\mathrm{t}, J=6.9,3 \mathrm{H}) ;{ }^{13} \mathrm{C} \mathrm{NMR}\left(125 \mathrm{MHz}, \mathrm{CDCl}_{3}\right)$ 134.1, 129.0, 63.8, 56.0, 50.0, 45.2, 34.1, 32.3, 31.9, 29.59, 29.56, 29.50, 29.46, 29.3, 29.1, 22.6, 22.1, 14.1; IR (film) 3336 (br), 2925, $1462 \mathrm{~cm}^{-1}$; HRMS (ESI) calcd for $\mathrm{C}_{22} \mathrm{H}_{44} \mathrm{NO}\left(\mathrm{M}+\mathrm{H}^{+}\right)$338.3423, found: 338.2430 . 


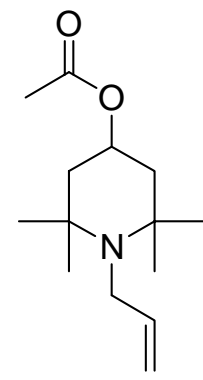

4-Acetoxy-1-(2-propenyl)-2,2,6,6-tetramethylpiperidine (3a). To a solution of amine S1 (555 $\mathrm{mg}, 2.81 \mathrm{mmol})$ and pyridine $(455 \mu \mathrm{L}, 5.62 \mathrm{mmol})$ in $\mathrm{CH}_{2} \mathrm{Cl}_{2}(5 \mathrm{~mL})$ was added $\mathrm{Ac}_{2} \mathrm{O}(530 \mu \mathrm{L}$, $5.6 \mathrm{mmol}$ ). The resultant solution was maintained at ambient temperature for $15 \mathrm{~h}$. After this period, the excess reagents were quenched by the addition of saturated aqueous $\mathrm{NaHCO}_{3}(5 \mathrm{~mL})$. The mixture was extracted with $\mathrm{CH}_{2} \mathrm{Cl}_{2}(3 \times 5 \mathrm{~mL})$. The combined organics were dried over $\mathrm{Na}_{2} \mathrm{SO}_{4}$ and concentrated under reduced pressure. The resultant oil was purified by flash chromatography $\left(\mathrm{SiO}_{2}, 10: 90\right.$ ethyl acetate:hexane) to provide $350 \mathrm{mg}(52 \%)$ of 3a as a colorless oil. ${ }^{1} \mathrm{H}$ NMR (500 MHz, $\left.\mathrm{CDCl}_{3}\right) 5.83$ (tdd, $\left.J=5.0,10.1,17.1,1 \mathrm{H}\right), 5.11-5.17$ (m, $\left.1 \mathrm{H}\right), 5.07$ (tt, $J=4.1,11.6,1 \mathrm{H}), 4.91-4.95$ (m, $1 \mathrm{H}), 3.12-3.15$ (m, $2 \mathrm{H}), 3.02$ (s, $3 \mathrm{H}), 1.79-1.84$ (m, $2 \mathrm{H})$, 1.47 (t, $J=11.6,2 \mathrm{H}), 1.079$ (s, $6 \mathrm{H}), 1.076$ (s, $6 \mathrm{H}) ;{ }^{13} \mathrm{C}\left(125 \mathrm{MHz}, \mathrm{CDCl}_{3}\right)$ 142.7, 112.9, 67.7, 56.0, 45.90, 45.85, 33.7, 22.3, 21.5; IR (film) 2968, 1738, $1244 \mathrm{~cm}^{-1}$; HRMS (ESI), calcd for $\mathrm{C}_{14} \mathrm{H}_{26} \mathrm{NO}_{2}\left(\mathrm{M}+\mathrm{H}^{+}\right)$240.1964, found: 240.1960 .

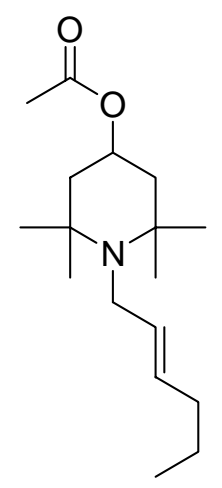

4-Hydroxy-1-(2-hexenyl)-2,2,6,6-tetramethylpiperidine (3b). To a solution of amine 7 (103 $\mathrm{mg}, 0.430 \mathrm{mmol})$, DMAP $(1 \mathrm{mg})$ and pyridine $(70 \mu \mathrm{L}, 0.9 \mathrm{mmol})$ in $\mathrm{CH}_{2} \mathrm{Cl}_{2}(2 \mathrm{~mL})$ was added $\mathrm{Ac}_{2} \mathrm{O}(80 \mu \mathrm{L}, 0.9 \mathrm{mmol})$. The resultant solution was maintained at ambient temperature for $24 \mathrm{~h}$ and then the excess reagents were quenched by addition of saturated aqueous $\mathrm{NaHCO}_{3}(3 \mathrm{~mL})$. 
The mixture was extracted with $\mathrm{CH}_{2} \mathrm{Cl}_{2}(3 \times 10 \mathrm{~mL})$ and the combined organics were dried over $\mathrm{Na}_{2} \mathrm{SO}_{4}$. The organic solution was concentrated under reduced pressure and the residue was purified by flash chromatography $\left(\mathrm{SiO}_{2}, \quad 5: 95\right.$ ethyl acetate:hexanes $\rightarrow$ 60:40 ethyl acetate:hexanes) to yield $99 \mathrm{mg}(82 \%)$ of $\mathbf{3 b}$ as a slightly yellow oil. ${ }^{1} \mathrm{H}$ NMR $(500 \mathrm{MHz}$, $\left.\mathrm{CDCl}_{3}\right)$ 5.35-5.55 (m, $\left.2 \mathrm{H}\right), 5.06$ (tt, $\left.J=4.1,11.6,1 \mathrm{H}\right), 3.08(\mathrm{~d}, J=4.4,2 \mathrm{H}), 2.02(\mathrm{~s}, 3 \mathrm{H}), 1.95$ (dd, $J=6.7,13.4,2$ H), 1.76-1.83 (m, 2 H), 1.46 (t, $J=11.7,2$ H), 1.30-1.41 (m, 2 H), 1.09 (s, 6 H), $1.07(\mathrm{~s}, 6 \mathrm{H}), 0.87(\mathrm{t}, J=7.4,3 \mathrm{H}) ;{ }^{13} \mathrm{C} \mathrm{NMR}\left(125 \mathrm{MHz}, \mathrm{CDCl}_{3}\right)$ 170.7, 134.3, 128.9, 67.8, 56.0, 45.9, 45.2, 34.4, 33.8, 22.6, 22.3, 21.4, 13.6; IR (film) 2965, 1738, $1244 \mathrm{~cm}^{-1}$; HRMS (ESI) Calcd for $\mathrm{C}_{17} \mathrm{H}_{32} \mathrm{NO}_{2}\left(\mathrm{M}+\mathrm{H}^{+}\right)$: 282.2433, Found: 282.2436.

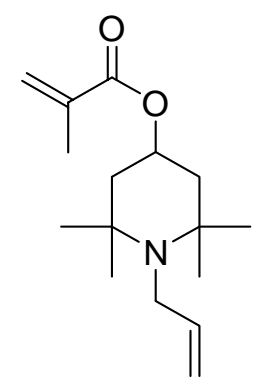

4-Methacryloyloxy-1-(2-propenyl)-2,2,6,6-tetramethylpiperidine (4a). To a chilled solution $\left(0{ }^{\circ} \mathrm{C}\right)$ of hydroxylamine $\mathbf{S 1}(501 \mathrm{mg}, 2.53 \mathrm{mmol})$, DMAP $(1 \mathrm{mg})$, pyridine $(225 \mu \mathrm{L}, 2.78 \mathrm{mmol})$ in $\mathrm{CH}_{2} \mathrm{Cl}_{2}(8 \mathrm{~mL})$ was added methacroyl chloride $(260 \mu \mathrm{L}, 2.66 \mathrm{mmol})$. The solution was allowed to warm to ambient temperature over $17 \mathrm{~h}$. After this period, the excess reagents were quenched by the addition of saturated aqueous $\mathrm{NaHCO}_{3}(5 \mathrm{~mL})$. The mixture was extracted with $\mathrm{CH}_{2} \mathrm{Cl}_{2}(3 \times 10 \mathrm{~mL})$. The combined organics were dried over $\mathrm{Na}_{2} \mathrm{SO}_{4}$ and concentrated under reduced pressure. The resultant oil was purified by flash chromatography $\left(\mathrm{SiO}_{2}, 10: 90\right.$ ethyl acetate:hexane) to provide $440 \mathrm{mg}(66 \%)$ of $4 \mathbf{a}$ as a colorless oil. ${ }^{1} \mathrm{H}$ NMR $\left(500 \mathrm{MHz}, \mathrm{CDCl}_{3}\right)$ 6.07-6.08 (m, $1 \mathrm{H}), 5.84$ (tdd, $J=5.0,10.1,17.1,1 \mathrm{H}), 5.52-5.54(\mathrm{~m}, 1 \mathrm{H}), 5.11-5.18(\mathrm{~m}, 2 \mathrm{H})$, 4.92-4.96 (m, $1 \mathrm{H}), 3.15$ (td, $J=1.8,4.8,2 \mathrm{H}), 1.82-1.94$ (m, $5 \mathrm{H}), 1.54$ (t, $J=11.8,2 \mathrm{H}), 1.10$ (s, $6 \mathrm{H}), 1.09$ (s, $6 \mathrm{H}) ;{ }^{13} \mathrm{C} \mathrm{NMR}\left(125 \mathrm{MHz}, \mathrm{CDCl}_{3}\right)$ 167.1, 142.7, 136.8, 125.0, 112.9, 68.1, 56.0, 
45.9, 45.8, 33.5, 22.5, 18.3; IR (film) 2968, 1715, $1165 \mathrm{~cm}^{-1}$; HRMS (ESI), calcd for $\mathrm{C}_{16} \mathrm{H}_{28} \mathrm{NO}_{2}$ $\left(\mathrm{M}+\mathrm{H}^{+}\right): 266.2120$, found: 266.2117.

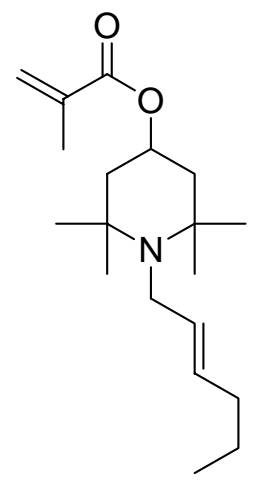

4-Methacryloyloxy-1-(E-2-hexenyl)-2,2,6,6-tetramethylpiperidine (4b). To a $0{ }^{\circ} \mathrm{C}$ solution of alcohol 7 (224 mg, $0.936 \mathrm{mmol}), \mathrm{NEt}_{3}(391 \mu \mathrm{L}, 2.81 \mathrm{mmol})$ and DMAP $(9 \mathrm{mg})$ in $\mathrm{CH}_{2} \mathrm{Cl}_{2}(3$ $\mathrm{mL})$ was added methacrylic acid anhydride $(279 \mu \mathrm{L}, 1.87 \mathrm{mmol})$. The solution was allowed to warm to ambient temperature over $14 \mathrm{~h}$. The excess anhydride was quenched by the addition of saturated aqueous $\mathrm{NaHCO}_{3}(5 \mathrm{~mL})$. The mixture was extracted with $\mathrm{CH}_{2} \mathrm{Cl}_{2}(3 \times 5 \mathrm{~mL})$ and the combined organics were dried over $\mathrm{Na}_{2} \mathrm{SO}_{4}$ and then concentrated under reduced pressure. The residue was purified by flash chromatography ( $\mathrm{SiO}_{2}, 20: 80$ ethyl acetate:hexanes) to provide 188 $\mathrm{mg}(66 \%)$ of $\mathbf{4 b}$ as a colorless oil. ${ }^{1} \mathrm{H}$ NMR $\left(500 \mathrm{MHz}, \mathrm{CDCl}_{3}\right) 6.01(\mathrm{~s}, 1 \mathrm{H}), 5.53$ (t, $J=1.53,1$ H), 5.36-5.50 (m, 2 H), 5.13 (tt, $J=4.2,11.5,1$ H) 3.08 (d, $J=4.8 \mathrm{~Hz}, 2 \mathrm{H}), 1.95$ (dd, $J=6.9$, 13.1, 2 H), 1.92 (s, 3 H), 1.84 (dd, $J=4.1,12.2,2$ H), 1.53 (t, $J=11.6,2$ H), 1.31-1.40 (m, 2 H), 1.10 (s, $6 \mathrm{H}), 1.09$ (s, $6 \mathrm{H}), 0.87(\mathrm{t}, J=7.3,3 \mathrm{H}) ;{ }^{13} \mathrm{C} \mathrm{NMR}\left(125 \mathrm{MHz}, \mathrm{CDCl}_{3}\right)$ 167.1, 136.8, 134.2, 129.0, 125.0, 68.1, 56.0, 45.8, 45.2, 34.4, 33.7, 22.7, 22.4, 18.3, 13.7; IR (film) 2966, 1716, $1164 \mathrm{~cm}^{-1}$; HRMS (ESI), calcd for $\mathrm{C}_{19} \mathrm{H}_{34} \mathrm{NO}_{2}\left(\mathrm{M}+\mathrm{H}^{\dagger}\right)$ 308.2589, found: 308.2591 . 


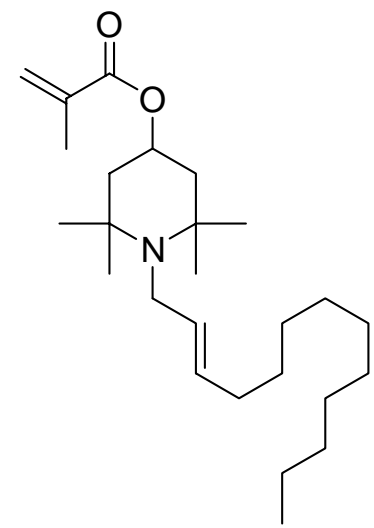

4-Methacryloyloxy-1-(E-2-tridecenyl)-2,2,6,6-tetramethylpiperidine (4c). To a solution of alcohol S2 (212 mg, $0.63 \mathrm{mmol})$, methacrylic acid (67 mg, $0.78 \mathrm{mmol})$ and DMAP (1 $\mathrm{mg})$ in $\mathrm{CH}_{2} \mathrm{Cl}_{2}(5 \mathrm{~mL})$ was added DCC $(157 \mathrm{mg}, 0.76 \mathrm{mmol})$. The solution was maintained at ambient temperature for $3 \mathrm{~h}$. The mixture was then concentrated under reduced pressure and the alcohol was dissolved in a solution of $20 \%$ ethyl acetate in hexanes $(5 \mathrm{~mL})$ and purified by flash chromatography $\left(\mathrm{SiO}_{2}, 20: 80\right.$ ethyl acetate:hexanes) to provide $204 \mathrm{mg}(78 \%)$ of 4c as a slightly yellow oil. ${ }^{1} \mathrm{H}$ NMR (500 MHz, $\left.\mathrm{CDCl}_{3}\right) 6.07$ (s, $\left.1 \mathrm{H}\right), 5.51-5.54$ (m, $\left.1 \mathrm{H}\right), 5.36-5.50$ (m, $\left.2 \mathrm{H}\right)$, $5.14(\mathrm{tt}, J=4.1,11.5,1 \mathrm{H}), 3.09(\mathrm{~d}, J=4.4,1 \mathrm{H}), 1.94-1.99(\mathrm{~m}, 2 \mathrm{H}), 1.93(\mathrm{~s}, 3 \mathrm{H}), 1.82-1.88$ $(\mathrm{m}, 2 \mathrm{H}), 1.53(\mathrm{t}, J=11.6,2 \mathrm{H}), 1.20-1.36(\mathrm{~m}, 17 \mathrm{H}), 1.10(\mathrm{~s}, 6 \mathrm{H}), 1.09(\mathrm{~s}, 6 \mathrm{H}), 0.88(\mathrm{t}, J=6.9$, $3 \mathrm{H}) ;{ }^{13} \mathrm{C} \mathrm{NMR}\left(125 \mathrm{MHz}, \mathrm{CDCl}_{3}\right)$ 167.1, 136.8, 134.0, 129.2, 125.0, 68.2, 56.0, 45.8, 45.2, $33.7,32.3,31.9,29.63,29.60,29.53,29.50,29.3,29.2,22.7,22.5,18.3,14.1$; IR (film) 2925 , 1717, $1164 \mathrm{~cm}^{-1}$; HRMS (ESI), calcd for $\mathrm{C}_{26} \mathrm{H}_{48} \mathrm{NO}_{2}\left(\mathrm{M}+\mathrm{H}^{+}\right)$406.3685, found: 406.3684 .

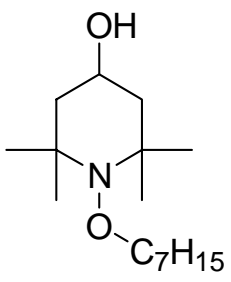

4-Hydroxy-1-(1-heptoxy)-2,2,6,6-tetramethylpiperidine

(S3). $\quad$ 4-Hydroxy-2,2,6,6tetramethylpiperinyloxy (720 mg, $4.2 \mathrm{mmol}), 1$-iodoheptane $(690 \mathrm{~mL}, 4.2 \mathrm{mmol})$ and tributyl tin hydride $(1.1 \mathrm{~mL}, 4.2 \mathrm{mmol})$ were dissolved in $\mathrm{C}_{6} \mathrm{H}_{6}(50 \mathrm{~mL})$ in a quartz flask. The solution was degassed by three freeze-pump-thaw cycles. The solution was irradiated with a Hanovia UV 
utility lamp (low pressure $\mathrm{Hg}$ ) for $5 \mathrm{~h}$. The mixture was concentrated under reduced pressure and the residue was purified by flash chromatography $\left(\mathrm{SiO}_{2}, 15: 85\right.$ ethyl acetate:hexanes) to yield $540 \mathrm{mg}(48 \%) \mathrm{S} 3$ as a colorless oil. ${ }^{1} \mathrm{H}$ NMR $\left(500 \mathrm{MHz}, \mathrm{CDCl}_{3}\right) 3.90-3.98(\mathrm{~m}, 1 \mathrm{H}), 3.71$ (t, $J=$ $6.3 \mathrm{~Hz}, 2 \mathrm{H}), 1.79$ (d, J = 9.5 Hz, 2 H), 1.40-1.55 (m, $4 \mathrm{H}), 1.25-1.39$ (m, $9 \mathrm{H}), 1.18$ (s, $6 \mathrm{H})$, 1.14 (s, $6 \mathrm{H}), 0.88$ (t, $J=7.0 \mathrm{~Hz}, 3 \mathrm{H}) ;{ }^{13} \mathrm{C}$ NMR $\left(125 \mathrm{MHz}, \mathrm{C}_{6} \mathrm{D}_{6}\right) 77.7,63.2,60.4,49.0,33.8$, 32.6, 30.2, 29.6, 27.2, 23.4, 21.5, 14.7 ; IR (film) 3359 (br), 2930, 1373, $1047 \mathrm{~cm}^{-1}$; HRMS (ESI) calcd for $\mathrm{C}_{16} \mathrm{H}_{34} \mathrm{O}_{2} \mathrm{~N}\left(\mathrm{M}+\mathrm{H}^{+}\right)$272.2589, found: 272.2586 .

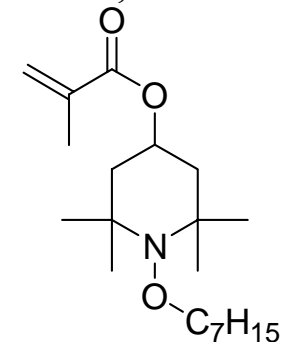

4-Methacryloyloxy-1-(1-heptoxy)-2,2,6,6-tetramethylpiperidine (9b). To a solution of alcohol S3 $(245 \mathrm{mg}, 0.903 \mathrm{mmol})$ and DMAP $(25 \mathrm{mg})$ in pyridine $(2 \mathrm{~mL})$ was added methacrylic acid anhydride $(150 \mu \mathrm{L}, 1.0 \mathrm{mmol})$. The resultant solution was maintained at ambient temperature for $22 \mathrm{~h}$. The excess reagents were quenched by addition of saturated aqueous $\mathrm{NaHCO}_{3}(3 \mathrm{~mL})$. The mixture was extracted with EtOAc $(2 \times 5 \mathrm{~mL})$ and the combined organics were washed with $1 \mathrm{M}$ $\mathrm{NaHSO}_{4}(3 \times 10 \mathrm{~mL})$. The combined organics were then dried over $\mathrm{MgSO}_{4}$ and concentrated under reduced pressure. The resulting residue was purified by flash chromatography $\left(\mathrm{SiO}_{2}, 5: 95\right.$ ethyl acetate:hexanes) to yield $69 \mathrm{mg}(22 \%) 9 \mathbf{b}$ as a colorless oil. ${ }^{1} \mathrm{H} \mathrm{NMR}\left(500 \mathrm{MHz}, \mathrm{CDCl}_{3}\right)$ $6.05(\mathrm{~s}, 1 \mathrm{H}), 5.52(\mathrm{~s}, 1 \mathrm{H}), 5.06(\mathrm{tt}, J=4.3,11.3,1 \mathrm{H}), 3.72(\mathrm{t}, J=6.7,2 \mathrm{H}), 1.91(\mathrm{~s}, 3 \mathrm{H}), 1.82-$ 1.88 (m, 2 H), 1.58 (t, $J=11.9,2$ H), 1.51 (td, $J=6.7,14.3,2 \mathrm{H}), 1.24-1.38(\mathrm{~m}, 8 \mathrm{H}), 1.19(\mathrm{~s}, 12$ $\mathrm{H}), 0.88(\mathrm{t}, J=6.9,3 \mathrm{H}) ;{ }^{13} \mathrm{C} \mathrm{NMR}\left(125 \mathrm{MHz}, \mathrm{CDCl}_{3}\right)$ 167.0, 136.7, 125.1, 77.0, 67.1, 59.9, 44.1, 33.1, 31.8, 29.4, 28.7, 26.4, 22.6, 20.9, 18.2, 14.1; IR (film) 2930, 1718, $1164 \mathrm{~cm}^{-1}$; HRMS (ESI), calcd for $\mathrm{C}_{20} \mathrm{H}_{38} \mathrm{NO}_{3}\left(\mathrm{M}+\mathrm{H}^{+}\right)$: 340.2852 , found: 340.2857 . 


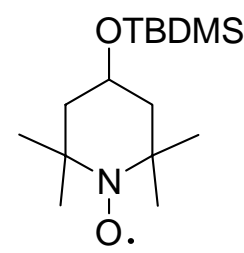

4-(tert-butyldimethylsilyloxy)-2,2,6,6-tetramethylpiperidinyloxy (14). To a solution of 4hydroxy-2,2,6,6-tetramethylpiperidinyloxy $(8.1 \mathrm{~g}, 47 \mathrm{mmol})$ and $\mathrm{NEt}_{3}(6.5 \mathrm{~mL}, 71 \mathrm{mmol})$ in $\mathrm{CH}_{2} \mathrm{Cl}_{2}(100 \mathrm{~mL})$ was added TBDMSCl $(8.5 \mathrm{~g}, 56 \mathrm{mmol})$. The resultant solution was maintained at ambient temperature for $17 \mathrm{~h}$. The reaction mixture was quenched by addition of saturated aqueous $\mathrm{NaHCO}_{3}(50 \mathrm{~mL})$. The mixture was partitioned and the aqueous layer was extracted with $\mathrm{CH}_{2} \mathrm{Cl}_{2}(3 \times 30 \mathrm{~mL})$. The combined organics were dried over $\mathrm{Na}_{2} \mathrm{SO}_{4}$ and concentrated under reduced pressure. The resultant oil was purified by flash chromatography $\left(\mathrm{SiO}_{2}: 20: 80\right.$ ethyl acetate:hexanes $\rightarrow 65: 35$ ethyl acetate:hexanes) to yield $5.3 \mathrm{~g}$ ( $65 \%$ yield) of 4-hydroxy2,2,6,6-tetramethylpiperdinyloxy as an orange solid and $4.5 \mathrm{~g}$ (33\% yield, 97\% yield brsm) of nitroxide 14 as a red solid. $\mathrm{mp} 32-34{ }^{\circ} \mathrm{C}$, IR (film) 2933, 1463, 1254, 1089, $836 \mathrm{~cm}^{-1}$; HRMS (ESI) Calcd for $\mathrm{C}_{15} \mathrm{H}_{32} \mathrm{NO}_{2} \mathrm{SiNa}\left(\mathrm{M}+\mathrm{Na}^{+}\right)$309.2100, Found: 309.2104; Anal. Calcd for $\mathrm{C}_{15} \mathrm{H}_{32} \mathrm{NO}_{2} \mathrm{Si}: \mathrm{C}, 62.88 ; \mathrm{H}, 11.26, \mathrm{~N}, 4.89$. Found: C, 63.00; H, 11.07; N, 4.64.

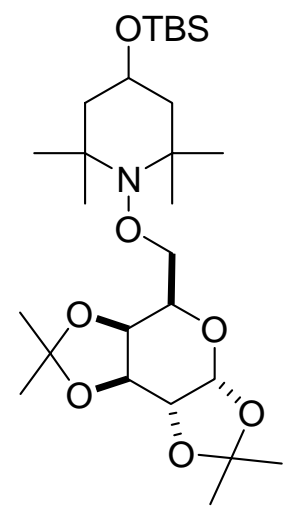

O-Diisopropylidene-galactose silyloxyhydroxylamine 15 . A solution of 4-(tertbutyldimethylsilyloxy)-2,2,6,6-tetramethylpiperidinyloxy (14) (1.56 g, $5.42 \mathrm{mmol})$, tributyltin hydride ( $870 \mu \mathrm{L}, 4.2 \mathrm{mmol})$, 5-iodo-diisopropylidene galactose (1.00 g, $2.70 \mathrm{mmol})$ and benzene $(50 \mathrm{~mL})$ in a quartz flask was degassed by four freeze-pump-thaw cycles. The degassed mixture was irradiated by a Hanovia UV utility lamp (low pressure $\mathrm{Hg}$ ) for $22 \mathrm{~h}$. The reaction mixture 
was concentrated under reduced pressure and the residue was purified by flash chromatography $\left(\mathrm{SiO}_{2}, 4: 96\right.$ ethyl acetate:hexane) to provide $1.02 \mathrm{~g}(71 \%)$ of $\mathbf{1 5}$ as a colorless oil. $[\alpha]_{23}^{\mathrm{D}}=-42.02$ (c 1.04, $\left.\mathrm{CHCl}_{3}\right) ;{ }^{1} \mathrm{H} \mathrm{NMR}\left(500 \mathrm{MHz}, \mathrm{CDCl}_{3}\right) 5.52(\mathrm{~d}, J=4.9,1 \mathrm{H}), 4.58(\mathrm{dd}, J=2.1,7.9,1 \mathrm{H})$, $4.28(\mathrm{dd}, J=2.2,4.9,1 \mathrm{H}), 4.24(\mathrm{~d}, J=7.9,1 \mathrm{H}), 3.84-4.00$ (m, 4 H), 1.60-1.67 (m, $2 \mathrm{H}), 1.54$ (s, 3 H), 1.44-1.54 (m, 2 H), 1.43 (s, 3 H), 1.33 (s, 3 H), 1.32 (s, 3 H), 1.20 (s, 3 H), 1.19 (s, 3 H), 1.13 (s, $3 \mathrm{H}), 1.12$ (s, $3 \mathrm{H}), 0.88$ (s, $9 \mathrm{H}), 0.05$ (s, $6 \mathrm{H}) ;{ }^{13} \mathrm{C} \mathrm{NMR}\left(125 \mathrm{MHz}, \mathrm{CDCl}_{3}\right)$ 109.1, 108.4, 96.3, 75.6, 71.5, 70.8, 70.6, 66.3, 63.9, 60.2, 60.0, 48.5, 33.1, 33.0, 26.1, 26.0, 25.9, 25.0, 24.4, 20.9, 18.2, -4.7 IR (film) 2934, 1378, $1074 \mathrm{~cm}^{-1}$; HRMS (ESI) Calcd for $\mathrm{C}_{27} \mathrm{H}_{51} \mathrm{NO}_{7} \mathrm{SiNa}$ $\left(\mathrm{M}+\mathrm{Na}^{+}\right)$552.3333, Found: 552.3344.

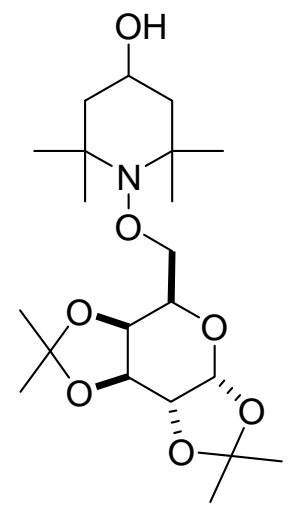

O-Diisopropylidene-galactose hydroxylamine (S4). To a chilled $\left(\begin{array}{ll}0 & \left.{ }^{\circ} \mathrm{C}\right) \\ \text { solution of }\end{array}\right.$ hydroxylamine $15(507 \mathrm{mg}, 0.958 \mathrm{mmol})$ in THF $(10 \mathrm{~mL})$ was added a $1.0 \mathrm{M}$ solution of TBAF $(1.9 \mathrm{~mL}, 1.9 \mathrm{mmol})$ in THF. The solution was allowed to warm to ambient temperature over $3 \mathrm{~h}$. After $3.3 \mathrm{~h}$, the reaction mixture was quenched by the addition of saturated aqueous $\mathrm{NaHCO}_{3}(10$ $\mathrm{mL})$. The mixture was extracted with $\mathrm{CH}_{2} \mathrm{Cl}_{2}(3 \times 10 \mathrm{~mL})$. The combined organics were dried over $\mathrm{Na}_{2} \mathrm{SO}_{4}$ and concentrated under reduced pressure. The resultant oil was purified by flash chromatography $\left(\mathrm{SiO}_{2}, 40: 60\right.$ ethyl acetate:hexane) to provide $355 \mathrm{mg}(89 \%)$ of $\mathbf{S} 4$ as a white solid. mp 114-116 ${ }^{\circ} \mathrm{C} ;[\alpha]_{23}^{\mathrm{D}}=-61.58\left(c 1.01, \mathrm{CHCl}_{3}\right) ;{ }^{1} \mathrm{H}$ NMR $\left(500 \mathrm{MHz}, \mathrm{C}_{6} \mathrm{D}_{6}\right) 5.51(\mathrm{~d}, J=$ 5.0, $1 \mathrm{H}), 4.49$ (dd, $J=2.2,7.9,1 \mathrm{H}), 4.22-4.28$ (m, 2 H), 4.18-4.22 (m, $1 \mathrm{H}), 4.16$ (dd, $J=2.3$, 5.0, $1 \mathrm{H}), 4.07$ (dd, $J=1.5,7.9,1 \mathrm{H}), 3.69-3.79$ (m, $1 \mathrm{H}), 1.60-1.71$ (m, $2 \mathrm{H}), 1.40-1.54$ (m, 8 H), 1.28-1.32 (m, 7 H), 1.15-1.20 (m, 9 H), 1.06 (s, $3 \mathrm{H}) ;{ }^{13} \mathrm{C}$ NMR (125 MHz, $\left.\mathrm{C}_{6} \mathrm{D}_{6}\right)$ 109.6, $108.8,97.2,76.9,72.5,71.6,71.5,67.3,63.2,60.8,60.6,49.1,33.8,33.5,26.6,25.4,24.9,21.5$; 
IR (film) 3394 (br), 2978, $1378 \mathrm{~cm}^{-1}$; HRMS (ESI) Calcd for $\mathrm{C}_{21} \mathrm{H}_{37} \mathrm{NO}_{7} \mathrm{Na}\left(\mathrm{M}+\mathrm{Na}^{+}\right)$438.2468, Found: 438.2467.

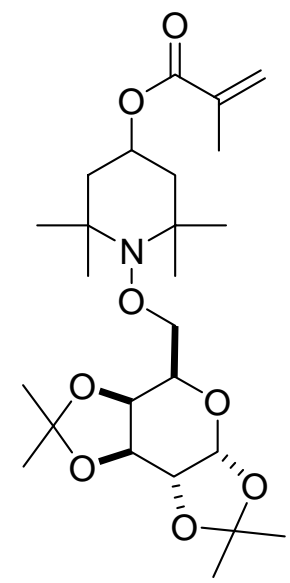

O-Diisopropylidene-galactose metacryloyloxyhydroxylamine 16. To a solution of hydroxylamine $\mathbf{S} 4(1.17 \mathrm{~g}, 2.82 \mathrm{mmol}), \mathrm{NEt}_{3}(786 \mu \mathrm{L}, 5.64 \mathrm{mmol})$ and DMAP $(1 \mathrm{mg})$ in $\mathrm{CH}_{2} \mathrm{Cl}_{2}$ $(10 \mathrm{~mL})$ was added methacroyl chloride $(386 \mu \mathrm{L}, 3.95 \mathrm{mmol})$. The resultant solution was maintained for $14 \mathrm{~h}$ at ambient temperature and then the excess reagents were quenched by addition of saturated aqueous $\mathrm{NaHCO}_{3}(10 \mathrm{~mL})$. The mixture was extracted with $\mathrm{CH}_{2} \mathrm{Cl}_{2}(3 \times 10$ $\mathrm{mL}$ ). The combined organics were dried over $\mathrm{Na}_{2} \mathrm{SO}_{4}$ and concentrated under reduced pressure. The resultant oil was purified by flash chromatography $\left(\mathrm{SiO}_{2}, 10: 90\right.$ ethyl acetate:hexane) to provide $1.08 \mathrm{~g}(79 \%)$ of $\mathbf{1 6}$ as a white solid. mp $83-85{ }^{\circ} \mathrm{C} ;[\alpha]_{23}^{\mathrm{D}}=-54.87\left(\right.$ c $\left.0.90, \mathrm{CHCl}_{3}\right) ;{ }^{1} \mathrm{H}$ NMR (500 MHz, $\left.{ }_{6} \mathrm{D}_{6}\right) 6.16(\mathrm{~s}, 1 \mathrm{H}), 5.51(\mathrm{~d}, J=5.0,1 \mathrm{H}), 5.22-5.25(\mathrm{~m}, 1 \mathrm{H}), 5.20(\operatorname{td}, J=4.3$, 11.7, 1 H), 4.49 (dd, $J=2.3,7.9,1$ H), 4.22-4.25 (m, 2 H), 4.17-4.20 (m, 1 H), 4.17 (dd, $J=2.3$, 7.9, $1 \mathrm{H}), 4.05(\mathrm{dd}, J=1.3,7.9,1 \mathrm{H}), 1.88(\mathrm{~s}, 3 \mathrm{H}), 1.82(\mathrm{td}, J=3.6,12.2,1 \mathrm{H}), 1.78(\mathrm{td}, J=3.6$, 12.2, $1 \mathrm{H}), 1.53-1.62(\mathrm{~m}, 2 \mathrm{H}), 1.47(\mathrm{~s}, 3 \mathrm{H}), 1.45(\mathrm{~s}, 3 \mathrm{H}), 1.25-1.27(\mathrm{~m}, 6 \mathrm{H}), 1.22(\mathrm{~s}, 3 \mathrm{H})$, $1.21(\mathrm{~s}, 3 \mathrm{H}), 1.16(\mathrm{~s}, 3 \mathrm{H}), 1.06(\mathrm{~s}, 3 \mathrm{H}) ;{ }^{13} \mathrm{C} \mathrm{NMR}\left(125 \mathrm{MHz}, \mathrm{CDCl}_{3}\right)$ 166.9, 137.7, 125.2, 109.6, 108.8, 97.2, 76.9, 72.4, 71.6, 71.5, 67.4, 67.1, 60.8, 60.6, 44.9, 44.8, 33.7, 33.4, 26.6, 25.3, 24.8, 21.3, 21.2, 18.8; IR (film) 2979, 1716, 1379, $1168 \mathrm{~cm}^{-1}$; HRMS (ESI) Calcd for $\mathrm{C}_{25} \mathrm{H}_{42} \mathrm{NO}_{8}$ $\left(\mathrm{M}+\mathrm{H}^{+}\right)$484.2910, Found: 484.2913. 


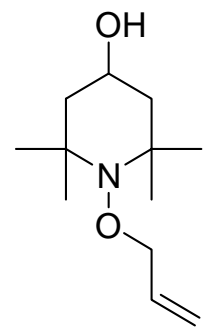

4-Hydroxy-1-(2-propenoxy)-2,2,6,6-tetramethylpiperidine (S5). A solution of 4-hydroxy2,2,6,6-tetramethylpiperidinyloxy (1.00 g, $5.80 \mathrm{mmol})$, tributyltin hydride $(1.56 \mathrm{~mL}, 5.80 \mathrm{mmol})$ and allyl bromide $(505 \mu \mathrm{L}, 5.80 \mathrm{mmol})$ and benzene $(50 \mathrm{~mL})$ in a quartz flask was degassed by three freeze-pump-thaw cycles. The degassed mixture was irradiated by a Hanovia UV utility lamp (low pressure $\mathrm{Hg}$ ) for $15 \mathrm{~h}$. The reaction mixture was concentrated under reduced pressure and the residue was purified by flash chromatography $\left(\mathrm{SiO}_{2}, 6: 94\right.$ ethyl acetate:hexanes $\rightarrow$ 15:85 ethyl acetate:hexane) to provide $472 \mathrm{mg}(38 \%)$ of S5 as a white solid. mp $58-60{ }^{\circ} \mathrm{C} ;{ }^{1} \mathrm{H} \mathrm{NMR}$ $\left(500 \mathrm{MHz}, \mathrm{CDCl}_{3}\right) 5.89$ (tdd, $\left.J=5.4,10.7,17.6,1 \mathrm{H}\right), 5.24-5.30(\mathrm{~m}, 1 \mathrm{H}), 5.11-5.15(\mathrm{~m}, 1 \mathrm{H})$, 4.27-4.30 (m, 2 H), 3.92-4.00 (m, 1 H), 1.78-1.84 (m, 2 H), 1.58 (br s, 1 H), 1.45 (t, J = 11.7, 2 $\mathrm{H}), 1.20(\mathrm{~s}, 6 \mathrm{H}), 1.16$ (s, $6 \mathrm{H}) ;{ }^{13} \mathrm{C} \mathrm{NMR}\left(125 \mathrm{MHz}, \mathrm{CDCl}_{3}\right)$ 133.9, 116.2, 78.4, 63.4, 60.0, 48.3, 33.0, 21.0; IR (film) 3267 (br), 2927, $1055 \mathrm{~cm}^{-1}$; HRMS (ESI) calcd for $\mathrm{C}_{12} \mathrm{H}_{24} \mathrm{NO}_{2}\left(\mathrm{M}+\mathrm{H}^{+}\right.$) 214.1807, found: 214.1802 .

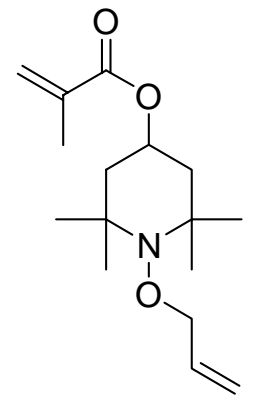

4-Methacryloyloxy-1-(2-propenoxy)-2,2,6,6-tetramethylpiperidine (17). To a solution of hydroxylamine S5 (55 mg, $0.26 \mathrm{mmol})$, DMAP (1 mg), $\mathrm{NEt}_{3}(73 \mu \mathrm{L}, 0.52 \mathrm{mmol})$ in $\mathrm{CH}_{2} \mathrm{Cl}_{2}(1$ $\mathrm{mL})$ was added methacrylic acid anhydride $(78 \mu \mathrm{L}, 52 \mathrm{mmol})$. The solution was maintained at ambient temperature for $9 \mathrm{~h}$. After this period, additional $\mathrm{NEt}_{3}(73 \mu \mathrm{L}, 0.52 \mathrm{mmol})$ and methacrylic acid anhydride $(78 \mu \mathrm{L}, 52 \mathrm{mmol})$ was added. The resultant solution was maintained 
for $4.5 \mathrm{~h}$ and then the excess reagents were quenched by the addition of saturated aqueous $\mathrm{NaHCO}_{3}(5 \mathrm{~mL})$. The mixture was extracted with $\mathrm{CH}_{2} \mathrm{Cl}_{2}(3 \times 5 \mathrm{~mL})$. The combined organics were dried over sodium sulfate and concentrated under reduced pressure. The resultant oil was purified by flash chromatography $\left(\mathrm{SiO}_{2}, 5: 95\right.$ ethyl acetate:hexane) to provide $55 \mathrm{mg}$ (76\%) of 17 as colorless oil. ${ }^{1} \mathrm{H}$ NMR (500 MHz, $\left.\mathrm{CDCl}_{3}\right) 6.06$ (s, $\left.1 \mathrm{H}\right), 5.89$ (tdd, $J=5.5,10.7,17.2,1$ H), $5.53(\mathrm{~s}, 1 \mathrm{H}), 5.28-5.24(\mathrm{~m}, 1 \mathrm{H}), 5.11-5.15(\mathrm{~m}, 1 \mathrm{H}), 5.08$ (tt, $J=4.4,11.3,1 \mathrm{H}), 4.27-4.31$ (m, 2 H), 1.92 (s, $3 \mathrm{H}), 1.85-1.90$ (m, $2 \mathrm{H}), 1.59$ (t, $J=11.9,2 \mathrm{H}), 1.22(\mathrm{~s}, 12 \mathrm{H}) ;{ }^{13} \mathrm{C}$ NMR (125 $\mathrm{MHz}, \mathrm{CDCl}_{3}$ ) 167.0, 136.6, 133.8, 125.2, 116.2, 78.4, 67.0, 60.0, 44.1, 33.0, 20.9, 18.2; IR (film) 2977, 1718, $1165 \mathrm{~cm}^{-1}$; HRMS (ESI) calcd for $\mathrm{C}_{16} \mathrm{H}_{28} \mathrm{NO}_{3}\left(\mathrm{M}+\mathrm{H}^{+}\right)$282.2069, found: 282.2064 .

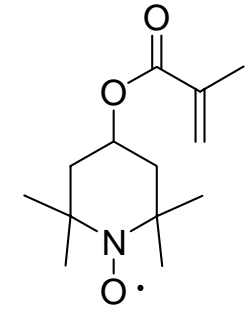

4-Methacroyl-2,2,6,6-tetramethylpiperidinyloxy (12b). To a solution of 4-hydroxy-2,2,6,6tetramethylpiperidinyloxy $(100 \mathrm{mg}, 0.58 \mathrm{mmol}), \mathrm{NEt}_{3}(170 \mu \mathrm{L}, 1.16 \mathrm{mmol})$ and DMAP $(1 \mathrm{mg})$ in $\mathrm{CH}_{2} \mathrm{Cl}_{2}(2 \mathrm{~mL})$ was added methacrylic acid anhydride $(172 \mathrm{~mL}, 1.16 \mathrm{mmol})$. The resulting solution was maintained at ambient temperature for $9 \mathrm{~h}$. After this period, additional $\mathrm{NEt}_{3}(170$ $\mu \mathrm{L}, 0.52 \mathrm{mmol})$ and methacrylic acid anhydride $(172 \mu \mathrm{L}, 1.16 \mathrm{mmol})$ was added. The resulting solution was maintained at ambient temperature for $4.5 \mathrm{~h}$ and then the excess reagents were quenched by addition of saturated aqueous $\mathrm{NaHCO}_{3}(5 \mathrm{~mL})$. The mixture was extracted with $\mathrm{CH}_{2} \mathrm{Cl}_{2}(3 \times 10 \mathrm{~mL})$. The combined organics were dried over $\mathrm{Na}_{2} \mathrm{SO}_{4}$ and concentrated under reduced pressure. The resultant oil was purified by flash chromatography $\left(\mathrm{SiO}_{2}, 5: 95\right.$ ethyl acetate:hexane) to provide $125 \mathrm{mg}(90 \%)$ of $\mathbf{1 2 b}$ as a pink solid. mp $86-87^{\circ} \mathrm{C}$, HRMS (ESI) Calcd for $\mathrm{C}_{13} \mathrm{H}_{22} \mathrm{NO}_{3} \mathrm{Na}\left(\mathrm{M}+\mathrm{Na}^{+}\right)$263.1497, Found: 263.1492; Anal. Calcd for $\mathrm{C}_{13} \mathrm{H}_{22} \mathrm{NO}_{3}: \mathrm{C}$, 64.97; H, 9.23; N, 5.83. Found: C, 64.79; H, 9.01; N, 5.62. 


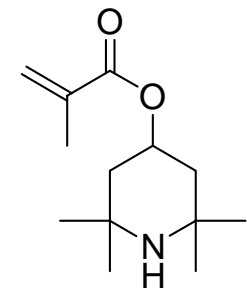

4-Methacryloyloxy-2,2,6,6-tetramethylpiperidine (S6). To a solution of 4-hydroxy-2,2,6,6tetramethylpiperidine ( $2.14 \mathrm{~g}, 13.6 \mathrm{mmol})$, DMAP $(166 \mathrm{mg}, 1.36 \mathrm{mmol})$ and pyridine $(1.43 \mathrm{~mL}$, $17.7 \mathrm{mmol})$ in $\mathrm{CH}_{2} \mathrm{Cl}_{2}(30 \mathrm{~mL})$ was added methacrylic acid anhydride $(2.43 \mathrm{~mL}, 16.3 \mathrm{mmol})$. The solution was maintained at ambient temperature for $12 \mathrm{~h}$ and then the excess reagents were quenched by the addition of saturated aqueous $\mathrm{NaHCO}_{3}(15 \mathrm{~mL})$. The mixture was extracted with $\mathrm{CH}_{2} \mathrm{Cl}_{2}(3 \times 15 \mathrm{~mL})$. The combined organics were dried over $\mathrm{Na}_{2} \mathrm{SO}_{4}$ and concentrated under reduced pressure. The resultant oil was purified by flash chromatography $\left(\mathrm{SiO}_{2}, 20: 80\right.$ ethyl acetate:hexane) to provide $1.32 \mathrm{mg}(43 \%)$ of $\mathbf{S 6}$ as white solid. mp $60-62{ }^{\circ} \mathrm{C} ;{ }^{1} \mathrm{H}$ NMR $\left(500 \mathrm{MHz}, \mathrm{CDCl}_{3}\right) 6.08(\mathrm{~s}, 1 \mathrm{H}), 5.53-5.55(\mathrm{~m}, 1 \mathrm{H}), 5.25(\mathrm{tt}, J=4.2,11.3,1 \mathrm{H}), 1.92-1.98(\mathrm{~m}$, $5 \mathrm{H}), 1.25$ (s, $6 \mathrm{H}), 1.20(\mathrm{t}, J=11.9,2 \mathrm{H}), 1.16(\mathrm{~s}, 6 \mathrm{H}) ;{ }^{13} \mathrm{C}$ NMR $\left(125 \mathrm{MHz}, \mathrm{CDCl}_{3}\right)$ 167.0, 136.8, 125.0, 69.1, 51.4, 43.8, 34.8, 29.1, 18.3; IR (film) 3312 (br), 2969, 1702, 1633, $1165 \mathrm{~cm}^{-1}$; Anal. Calcd for $\mathrm{C}_{13} \mathrm{H}_{23} \mathrm{NO}_{2}$ : C, 69.29; H, 10.29; N, 6.22. Found: C, 69.50; H, 10.21; N, 6.09. 


\section{Polymerizations}

\section{General Procedure for Polymerization. Preparation of hexenylamine template copolymer}

S7. Ethylene glycol dimethacrylate $(1.84 \mathrm{~mL}, 9.76 \mathrm{mmol}, 0.8$ equiv), methyl methacrylate (182 $\mu \mathrm{L}, 1.71 \mathrm{mmol}, 0.14$ equiv), amine $4 \mathbf{b}$ (188 $\mathrm{mg}, 0.61 \mathrm{mmol}, 0.05$ equiv), AIBN (20 mg, 0.1 mmol, 0.01 equiv) and $\mathrm{CH}_{3} \mathrm{CN}(2.25 \mathrm{~mL})$ were combined in a $20 \mathrm{~mL}$ vial. The solution was degassed for 5 min with argon and then sealed and placed in an $80{ }^{\circ} \mathrm{C}$ oil bath for $40 \mathrm{~h}$. After cooling to ambient temperature, the solid was crushed and placed in a Soxholet extractor and extracted with $\mathrm{MeOH}$ for $24 \mathrm{~h}$. The solid was then dried under vacuum to provide $3.73 \mathrm{~g}$ of polymer S7.

Blank Polymer S8: Following the general experimental amine S6 (225 mg, $1.00 \mathrm{mmol})$ was processed to provide $3.16 \mathrm{~g}$ of amino polymer $\mathbf{S 8}$.

Allyl Polymer S9: Following the general experimental amine 4a $(265 \mathrm{mg}, 1.00 \mathrm{mmol})$ was processed to provide $3.73 \mathrm{~g}$ of amino polymer S9.

Tridecenyl Polymer S10: Following the general experimental amine 4c (406 mg, $1.00 \mathrm{mmol}$ ) was processed to provide $3.81 \mathrm{~g}$ of amino polymer S10.

Diisopropylidene Galactose Polymer S11: Following the general experimental amine 16 (200 $\mathrm{mg}, 0.41 \mathrm{mmol}$ ) was processed to provide $1.63 \mathrm{~g}$ of amino polymer $\mathbf{S 1 1}$.

O-Heptyl Polymer S12: Following the general experimental amine $9 \mathbf{b}(118 \mathrm{mg}, 0.35 \mathrm{mmol})$ was processed to provide $1.32 \mathrm{~g}$ of amino polymer $\mathbf{S 1 2}$.

O-Allyl Polymer S13: Following the general experimental amine 17 (272 $\mathrm{mg}, 0.98 \mathrm{mmol})$ was processed to provide $2.78 \mathrm{~g}$ of amino polymer S13. 


\section{General Procedure for the Generation of Nitroxide Containing Polymers. Preparation of} hexenylamine templated nitroxide polymer S14. To a suspension of polymer S7 (2.03 g) in $\mathrm{CH}_{2} \mathrm{Cl}_{2}(10 \mathrm{~mL})$ was added $m$-CPBA $(0.986 \mathrm{~g}, 5.71 \mathrm{mmol})$. The mixture was allowed to stand for $18 \mathrm{~h}$ and then filtered and washed with $\mathrm{CH}_{2} \mathrm{Cl}_{2}(3 \times 10 \mathrm{~mL})$. The resulting pink solid was placed in a Soxholet extractor and extracted with $\mathrm{MeOH}$ for $24 \mathrm{~h}$. The solid was dried under vacuum to provide $2.53 \mathrm{~g}$ of templated nitroxide polymer $\mathbf{S 1 4}$ as a pink solid.

Blank Nitroxide Polymer S15: Following the general experimental, polymer S8 (2.93 g) was converted to polymer S15 (3.12 g).

Allyl Templated Polymer S16: Following the general experimental, polymer S9 (3.54 g) was converted to polymer S16 (3.12 g).

Tridecenyl Templated Polymer S17: Following the general experimental, polymer S10 (3.64 g) was converted to polymer S17 (4.35 g).

Galactose Templated Polymer S18: Following the general experimental, polymer S11 (1.55 g) was converted to polymer $\mathbf{S 1 8}(1.50 \mathrm{~g})$

O-Heptyl Templated Polymer S19: Following the general experimental, polymer S12 (1.00 g) was converted to polymer S19 $(1.00 \mathrm{~g})$.

O-Allyl Templated Polymer S20: Following the general experimental, polymer S13 ( $0.96 \mathrm{~g})$ was converted to polymer $\mathbf{S 2 0}(0.99 \mathrm{~g})$ 


\section{Oxidation Chemistry}

Table 1, Entry 1: Benzaldehyde. Following the general experimental (see text, Ref. 21), benzyl alcohol $(66 \mathrm{mg})$ was converted to benzaldehyde $(52 \mathrm{mg}, 80 \%$ yield).Data are in agreement with literature values. ${ }^{3}$

Table 1, Entry 2: Phenyl Ethyl Ketone. Following the general experimental (see text, Ref. 21), 1-phenylpropan-1-ol (83 mg) was converted to phenyl ethyl ketone (62 mg, $76 \%$ yield). Data are in agreement with literature values. ${ }^{4}$

Table 1, Entry 3: Decanal. Following the general experimental (see text, Ref. 21), 1-decanol (100 mg) was converted to Decanal (79 mg, $80 \%$ yield). Data are in agreement with literature values. $^{5}$

Table 1, Entry 4: 5-Phenyl Pentanal. See References 21-22 in the text.

Table 1, Entry 5: 2-Methyl-Dodecanal. Following the general experimental (see text, Ref. 21), 2-methyl-1-dodecanol (50 mg) was converted to 2-methyl-dodecanal (33 mg, $68 \%$ yield). Data are in agreement with literature values. ${ }^{6}$

${ }^{3}$ Abraham, R. J.; Chadwick, D. J.; Sancassan, F. Tetrahedron 1982, 38, 1485-1492.

${ }^{4}$ Wang, D.; Zhang, Z. Org. Lett. 2003, 5, 4645-4648.

${ }^{5}$ Velusamy, S.; Punniyamurthy, T. Org. Lett. 2004, 6, 217-220.

${ }^{6}$ Fischli, A. Helv. Chim. Acta 1978, 61, 2560-2578. 


\section{E. Representative EPR Spectra}

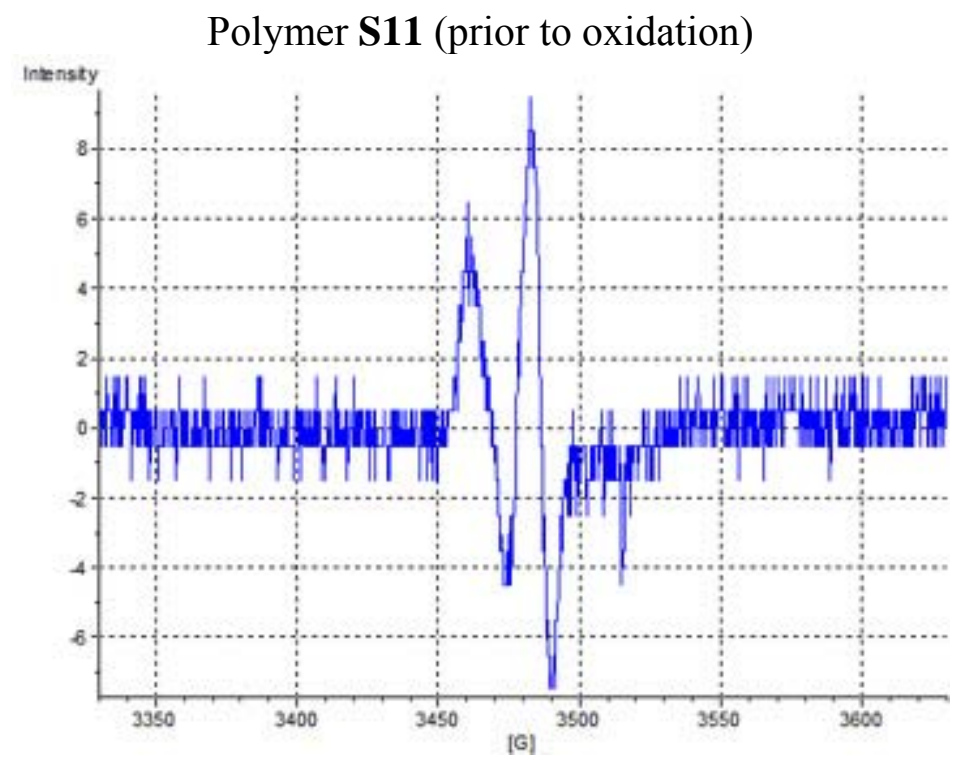

Polymer S18

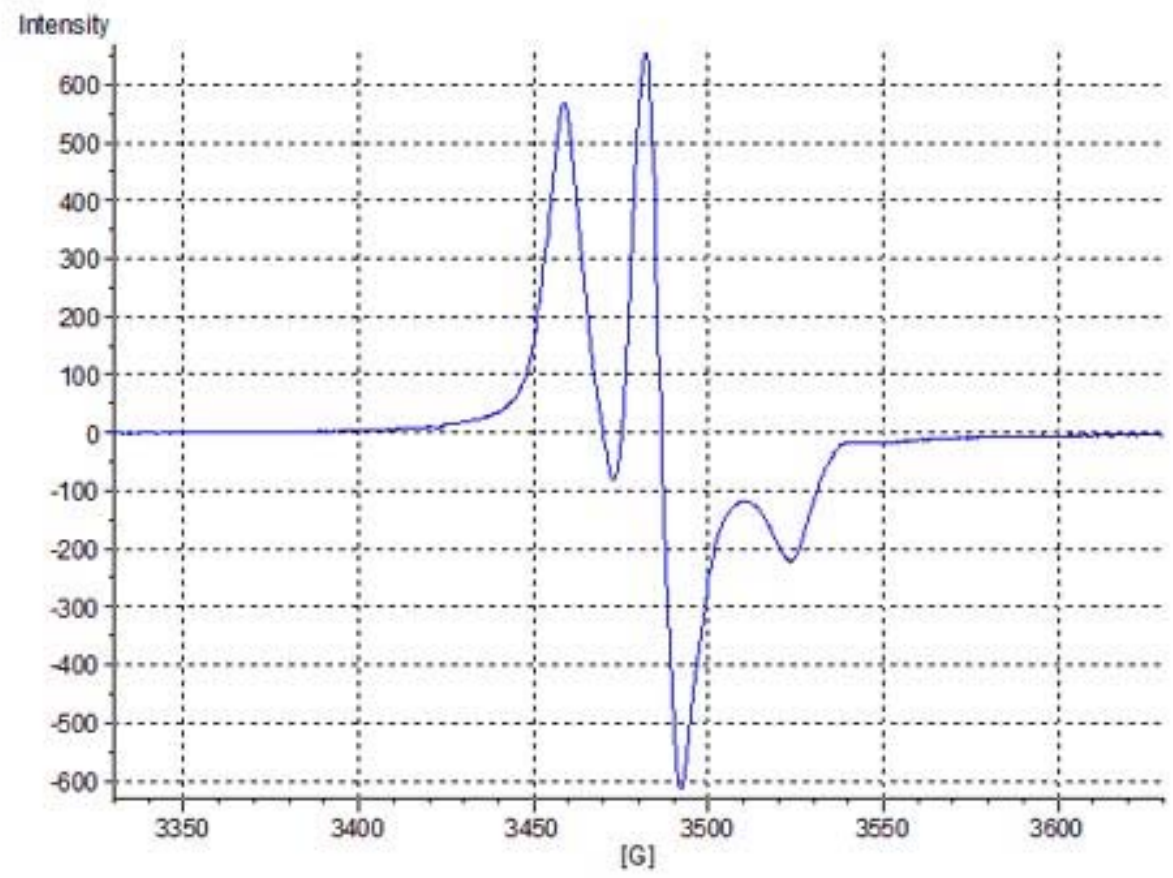




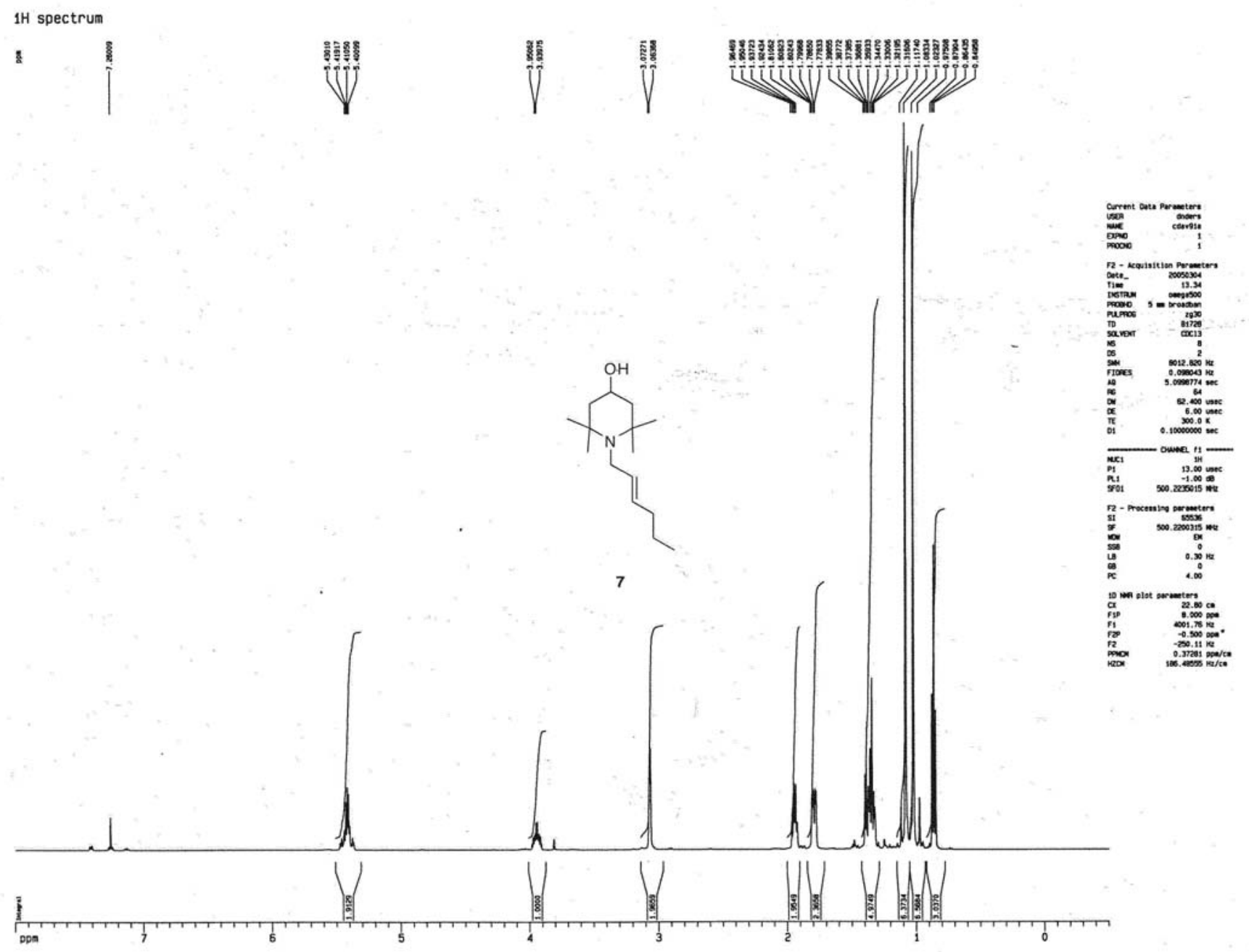


$13 \mathrm{C}$ spectrum with $1 \mathrm{H}$ decoupling

$$
8
$$
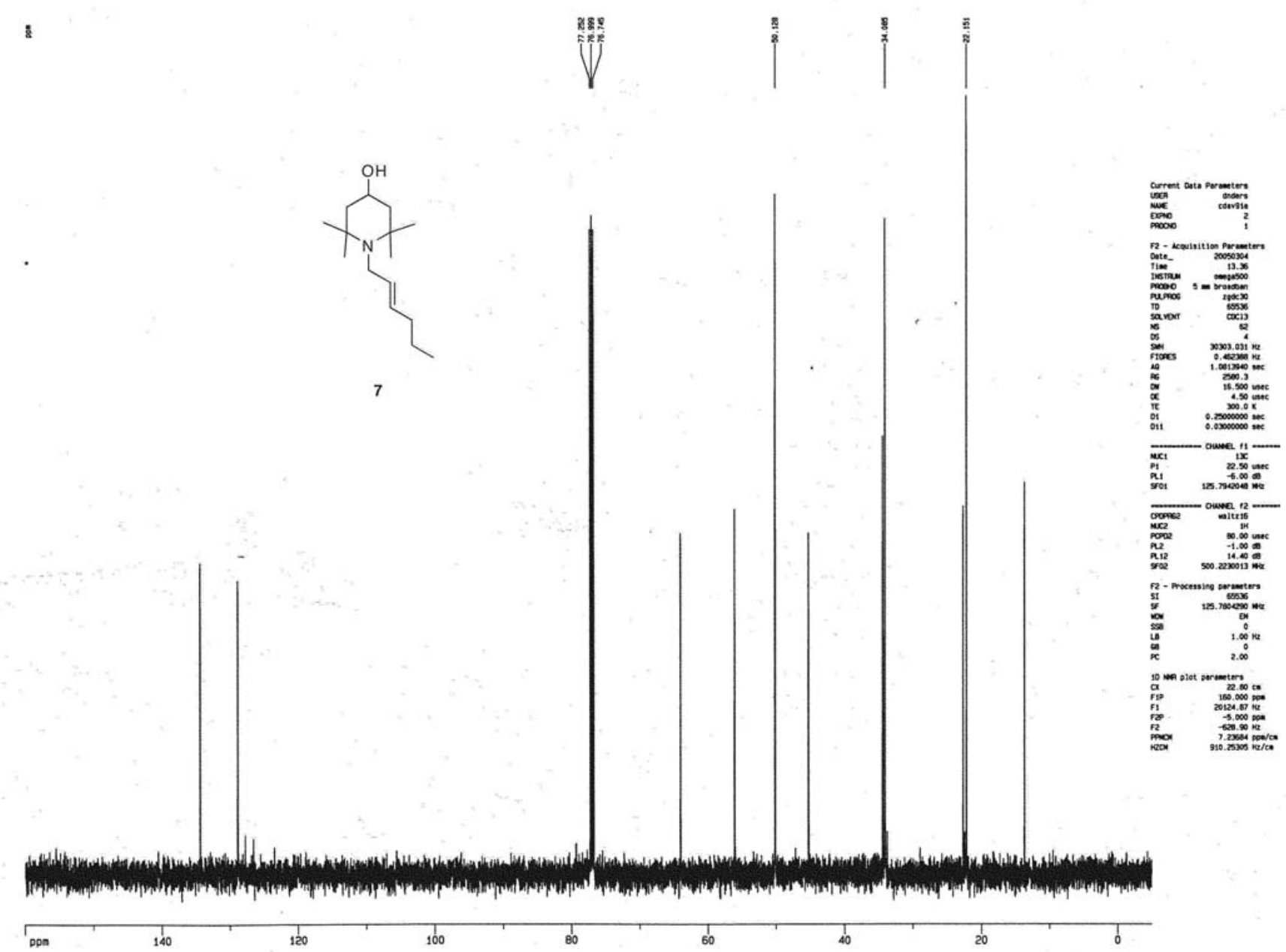


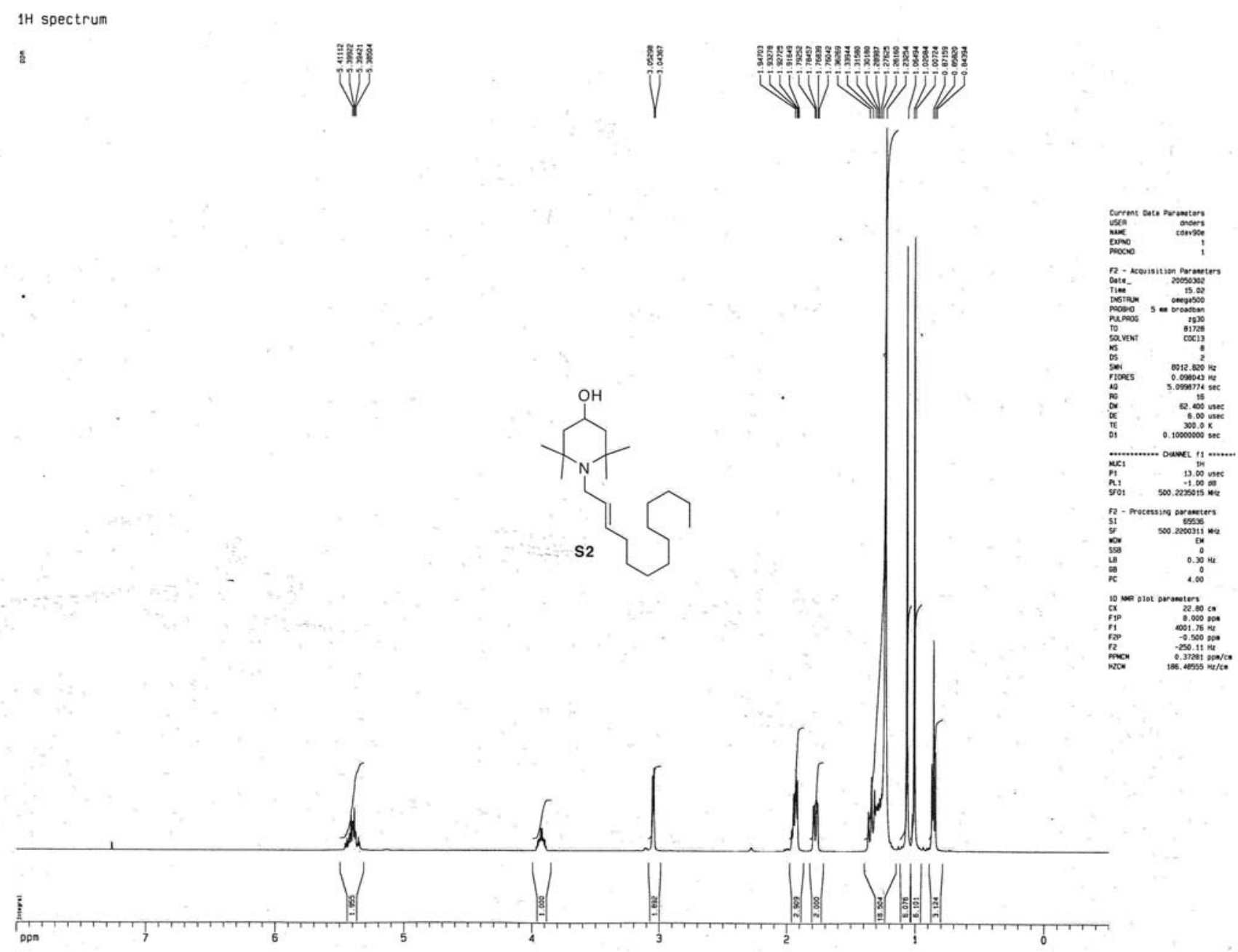


$13 C$ spectrum with $1 \mathrm{H}$ decoupling

\&
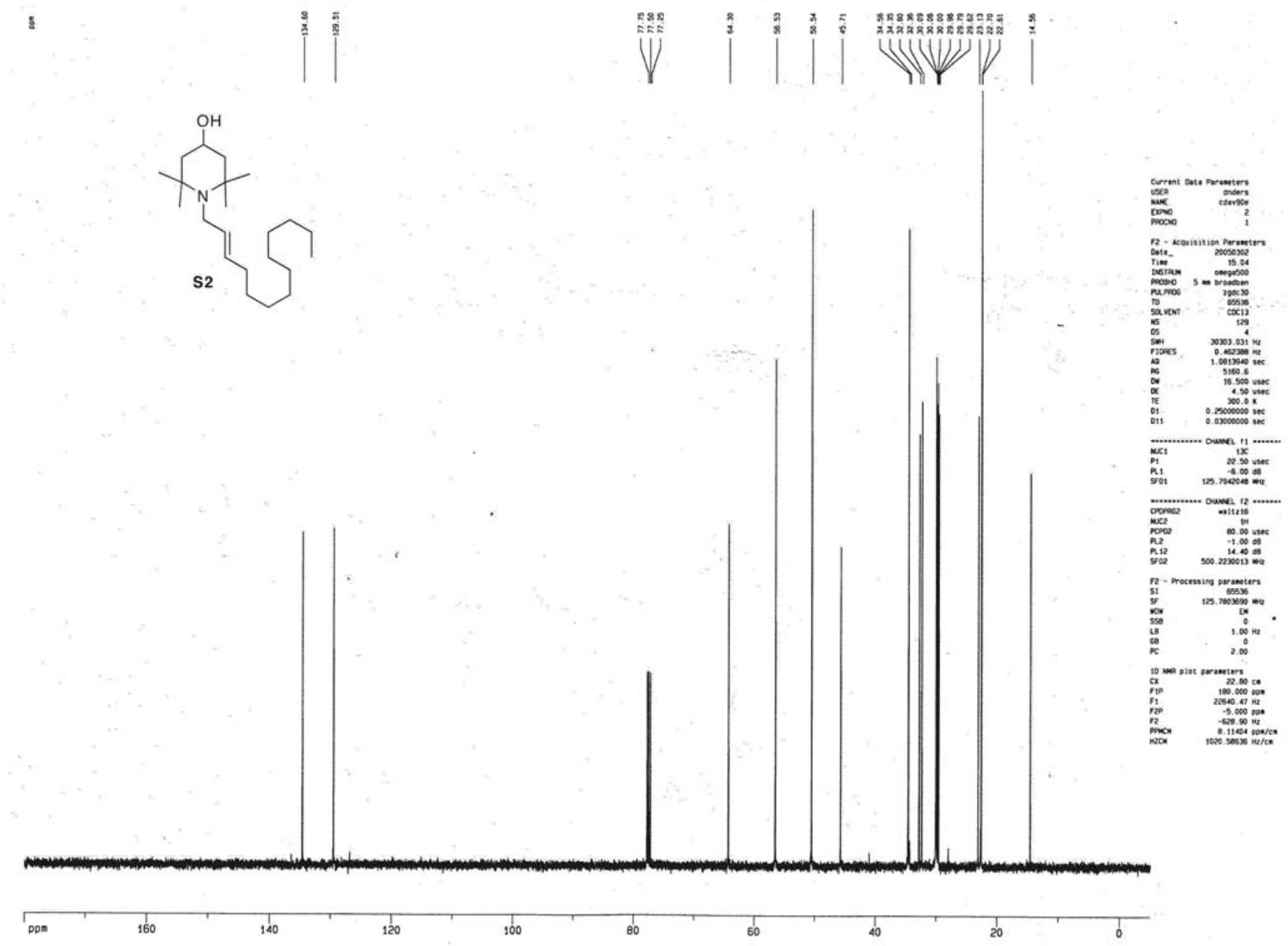
1H spectrum

s

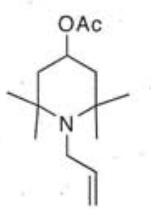

3a

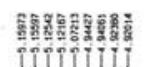

iviofiv

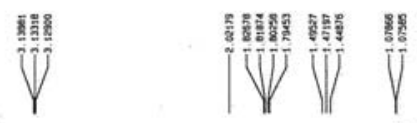

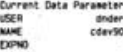

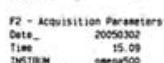

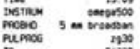

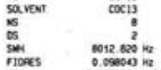

$$
\begin{aligned}
& \text { \$ }
\end{aligned}
$$

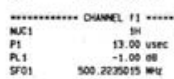

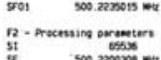

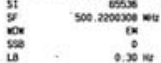

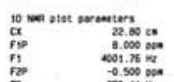


$13 \mathrm{C}$ spectrum with $1 \mathrm{H}$ decoupling

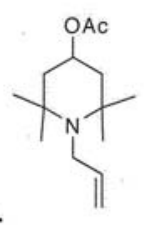

3a
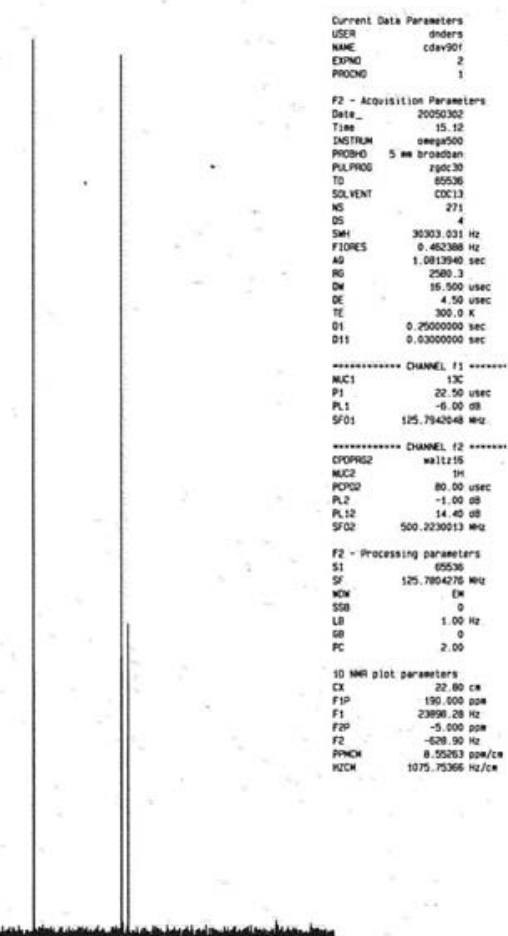

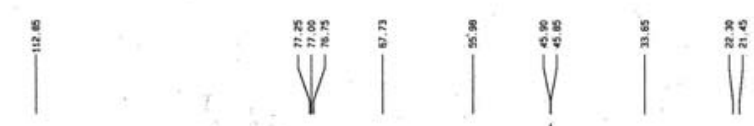
${ }_{50}^{1}$ 20 


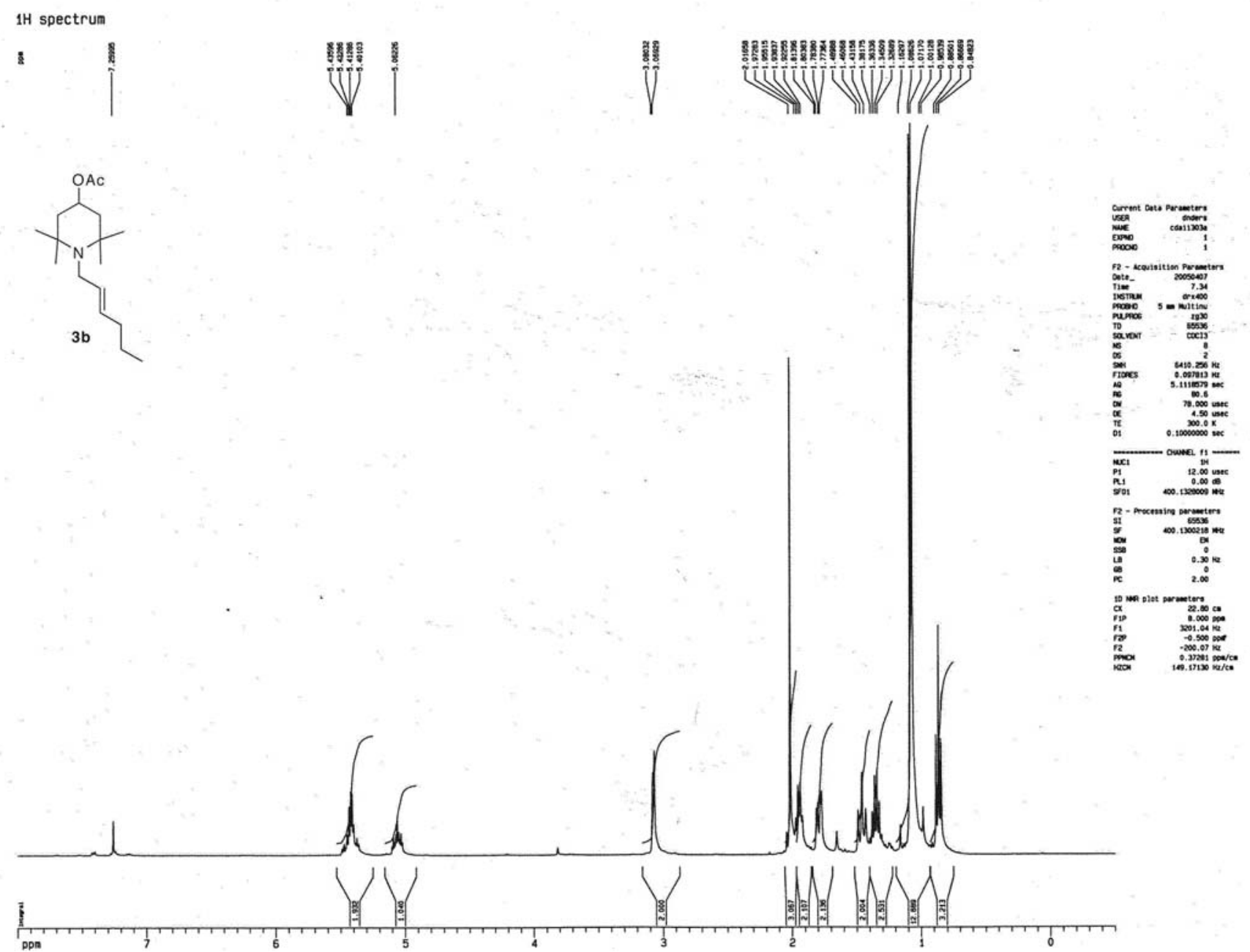


$13 \mathrm{C}$ spectrum with $1 \mathrm{H}$ decoupling

s
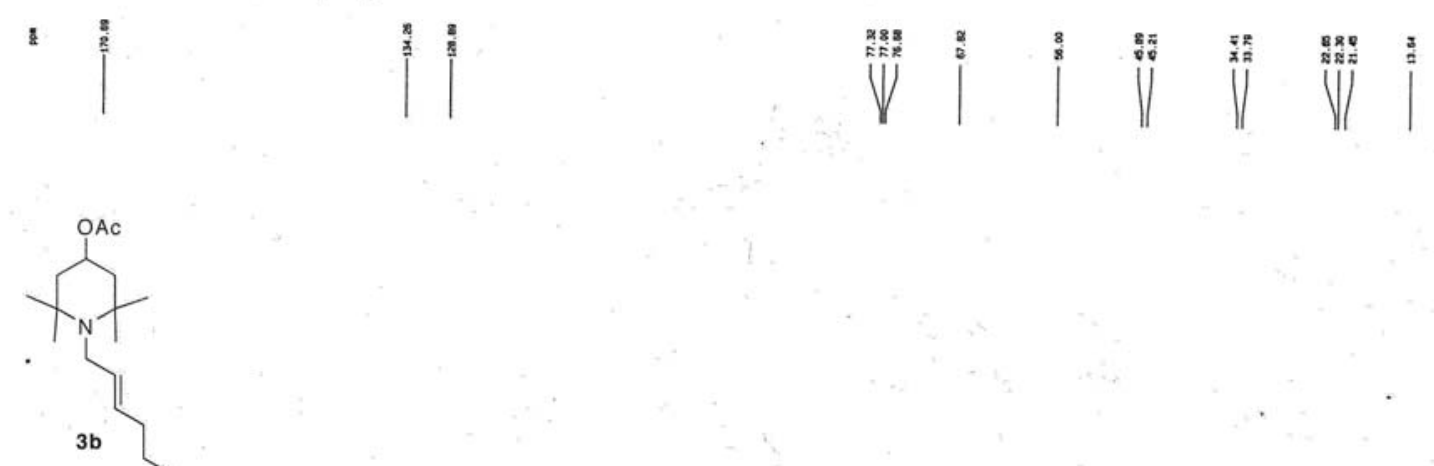

3b

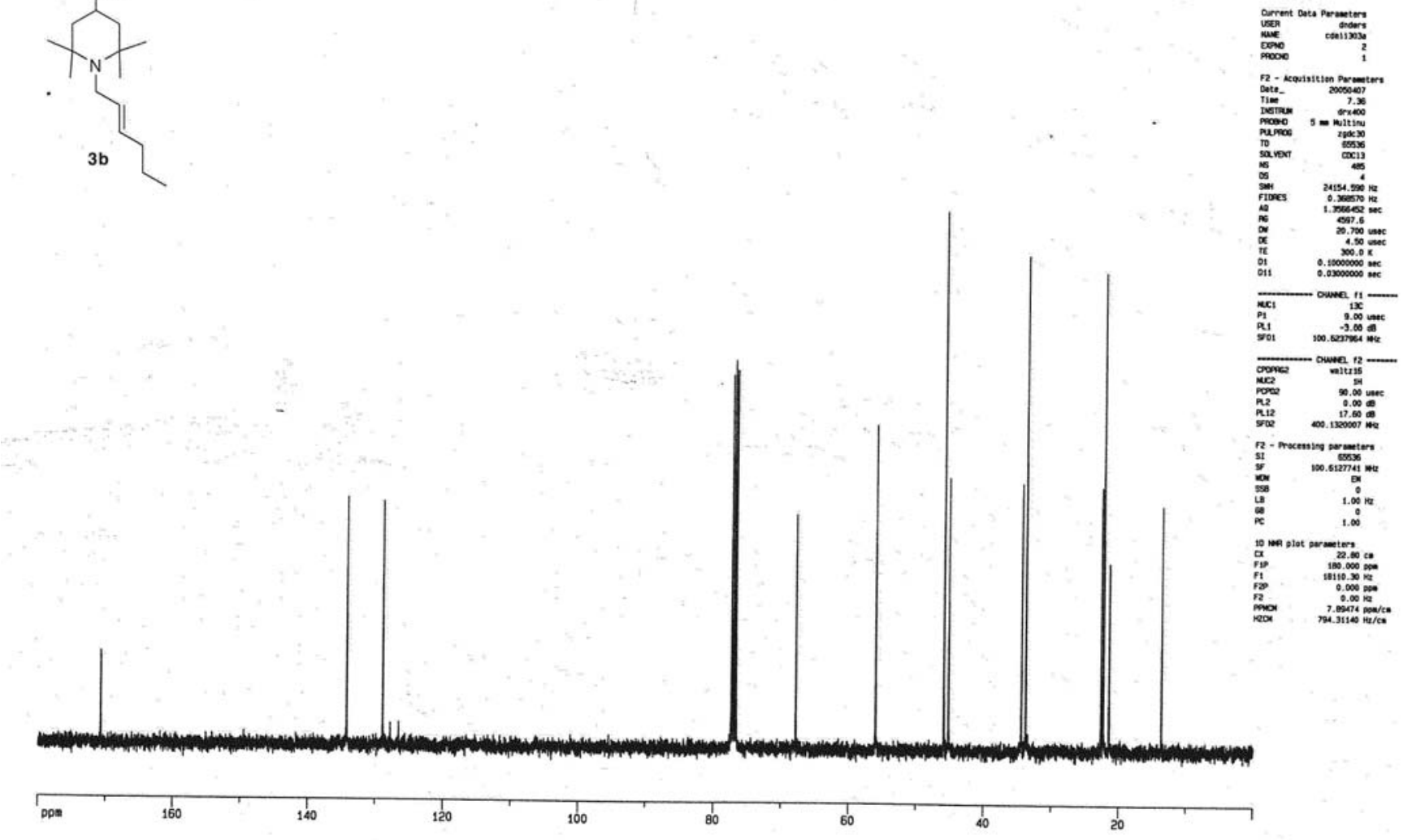


1H spectrum
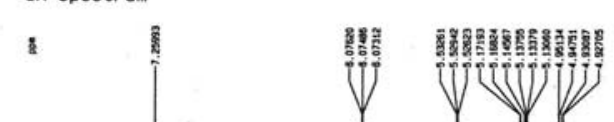

iv

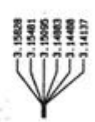
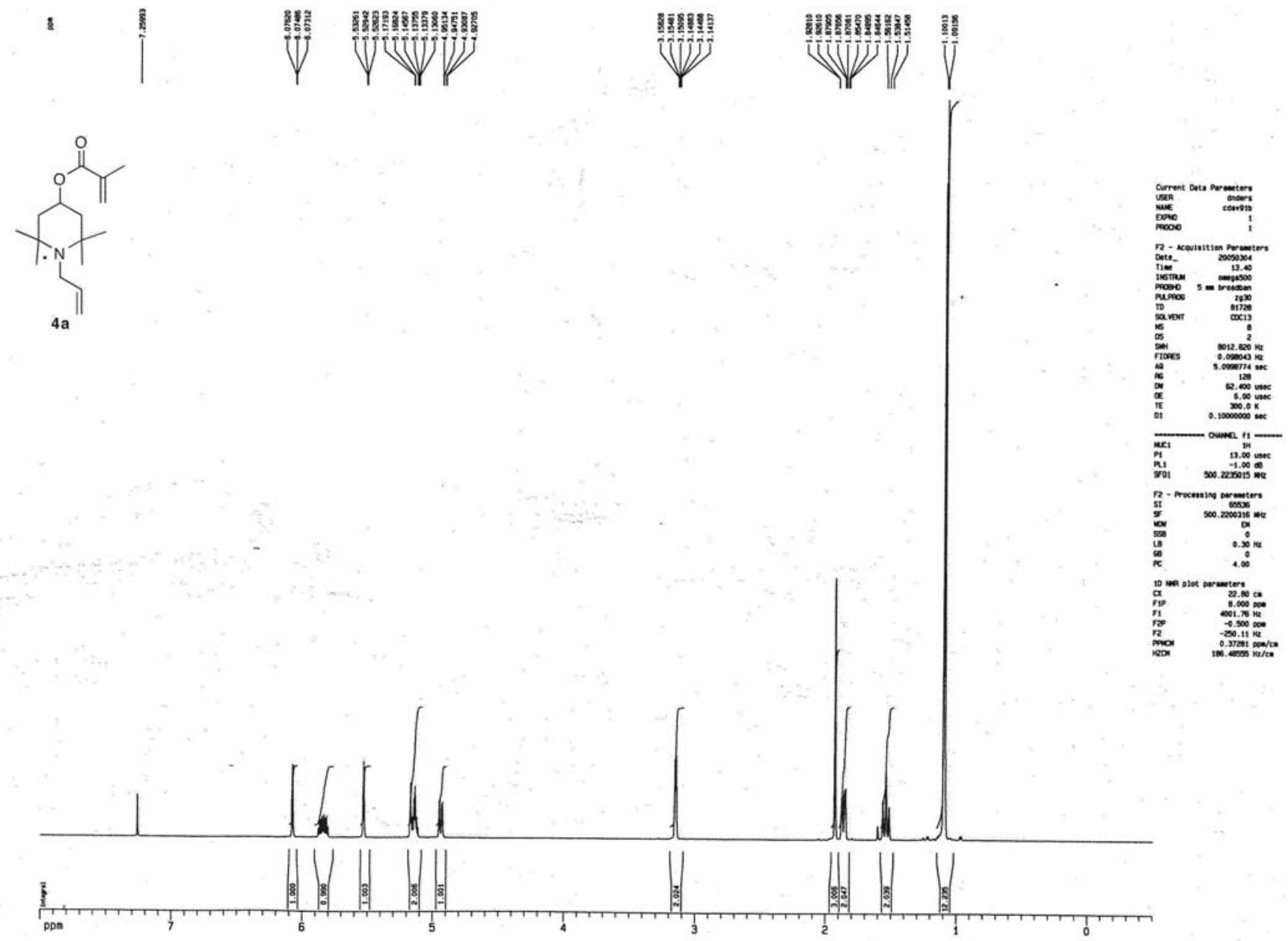
$13 \mathrm{C}$ spectrum with $1 \mathrm{H}$ decoupling

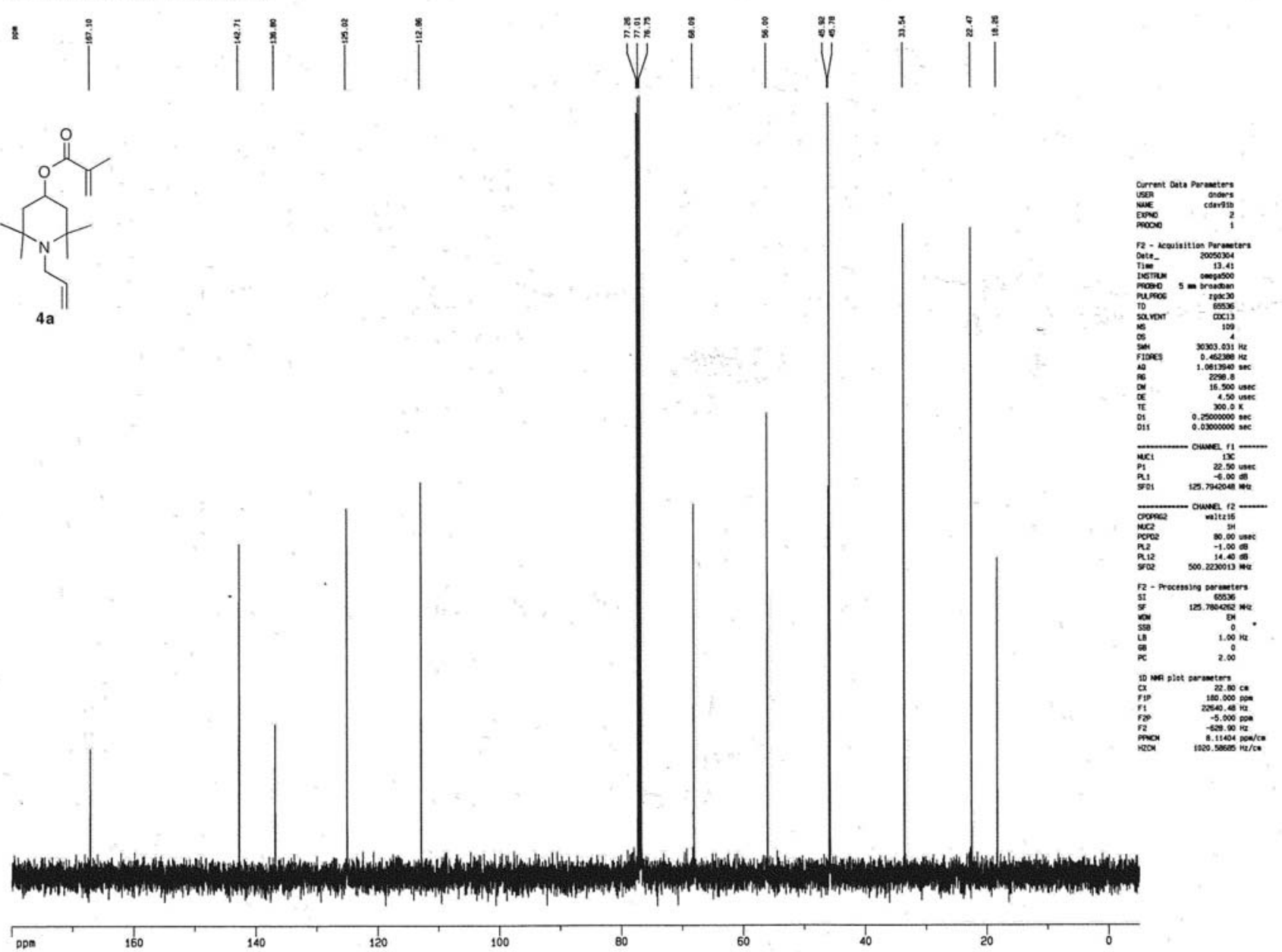




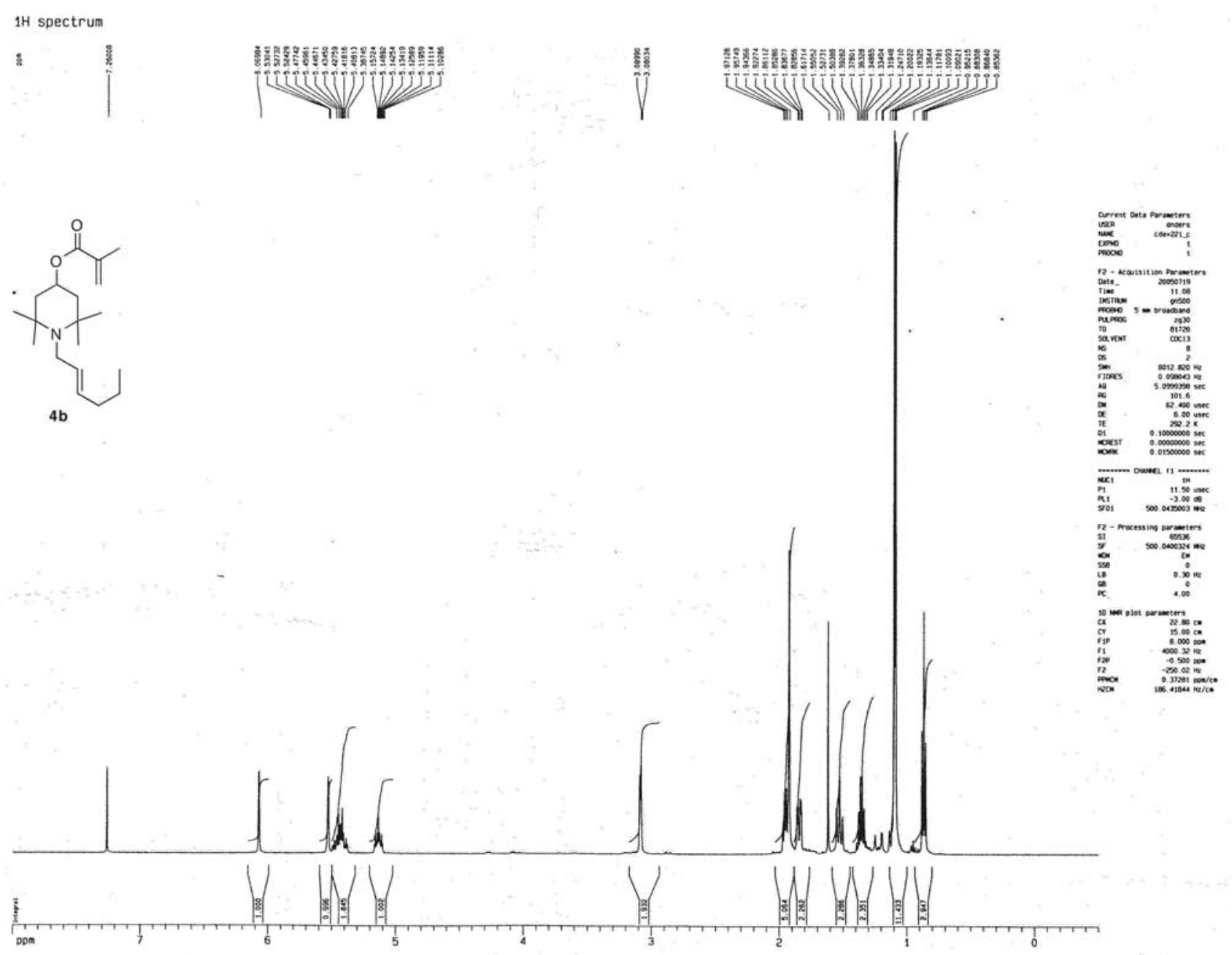


$13 \mathrm{C}$ spectrum with $1 \mathrm{H}$ decoupling

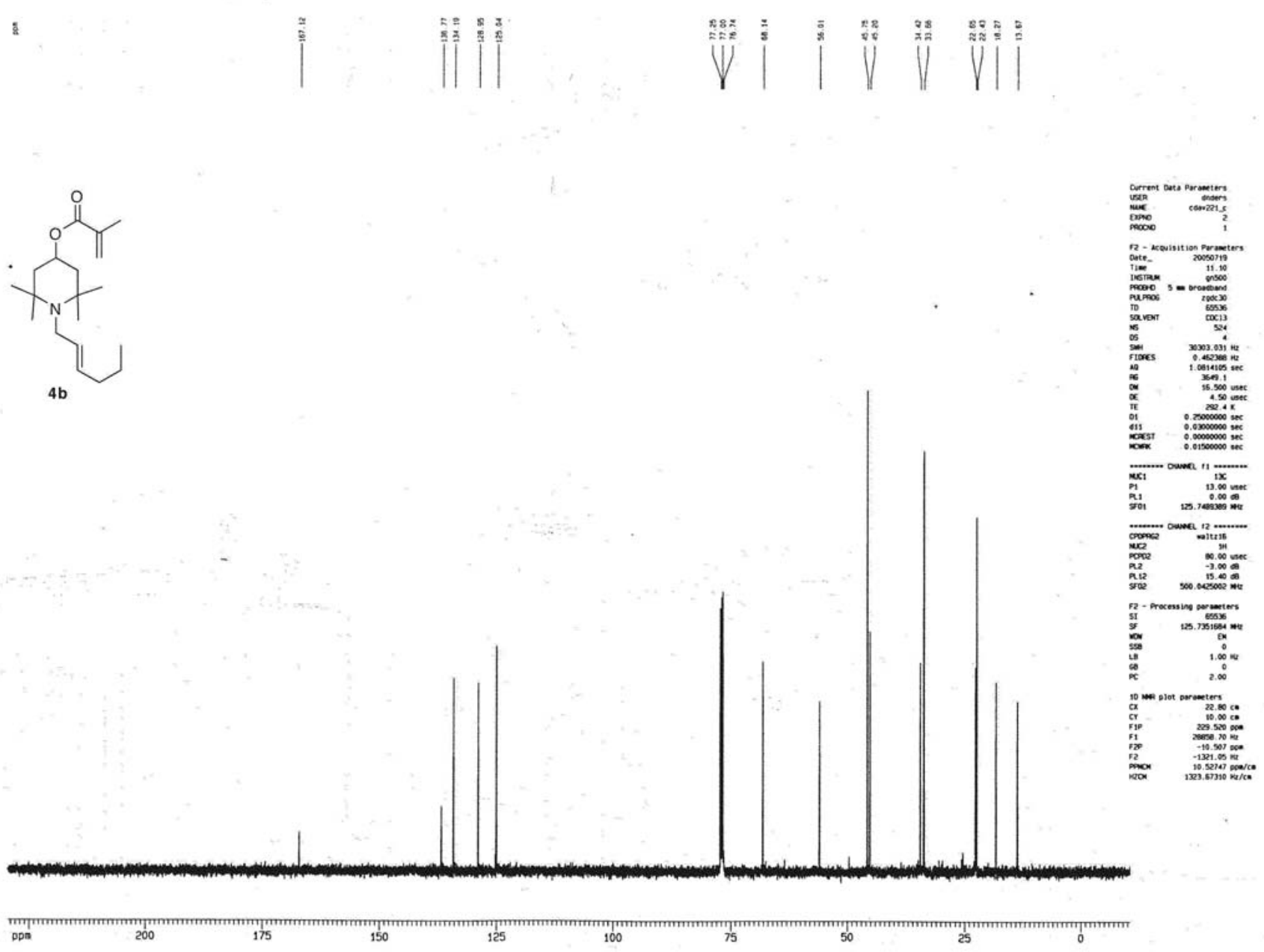


$1 \mathrm{H}$ spectrum
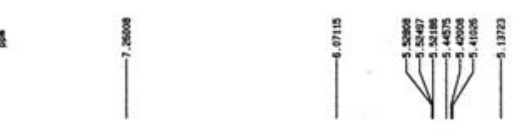

ขึำ

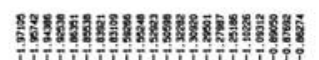

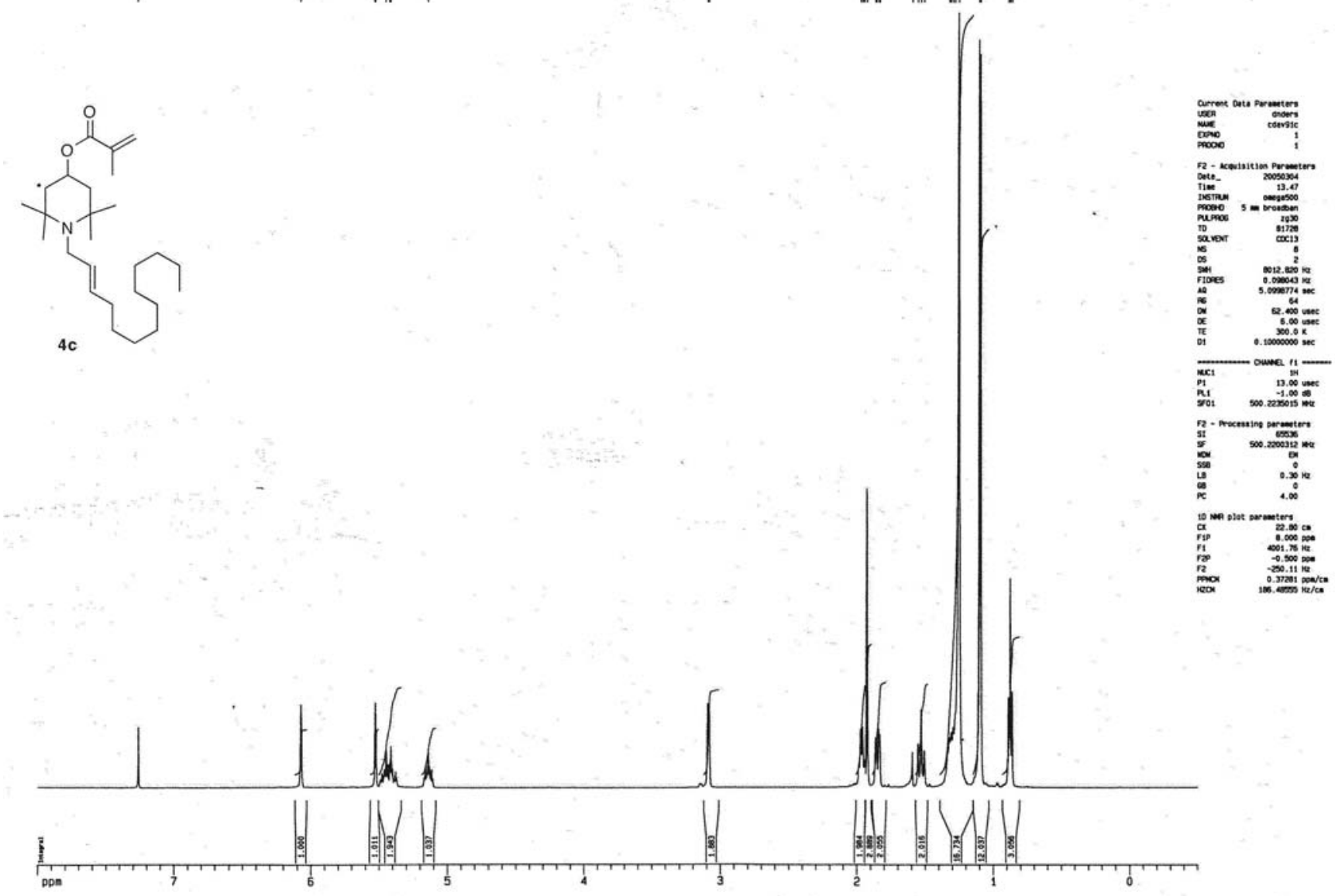


$13 \mathrm{C}$ spectrum with $1 \mathrm{H}$ decoupling

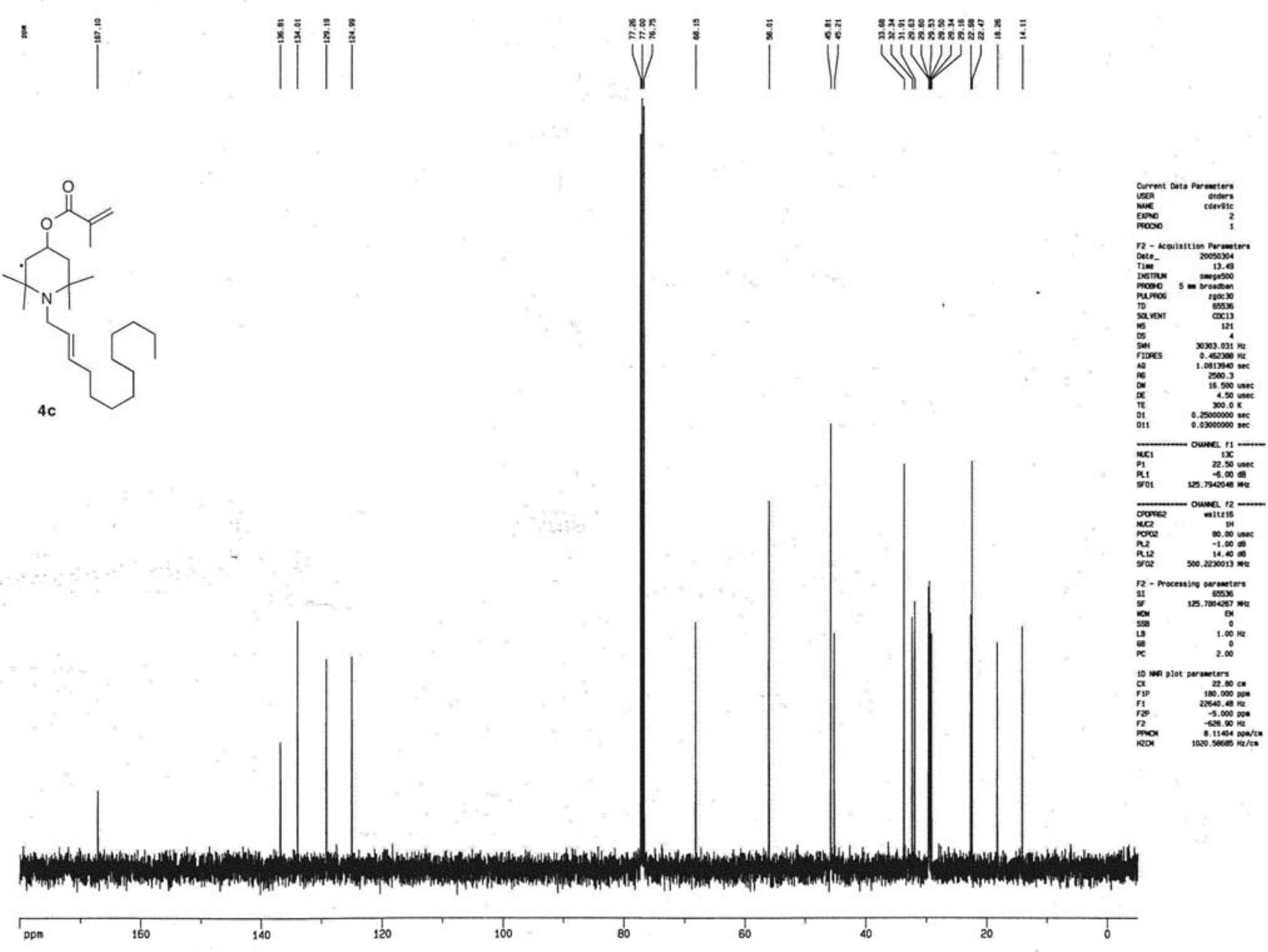




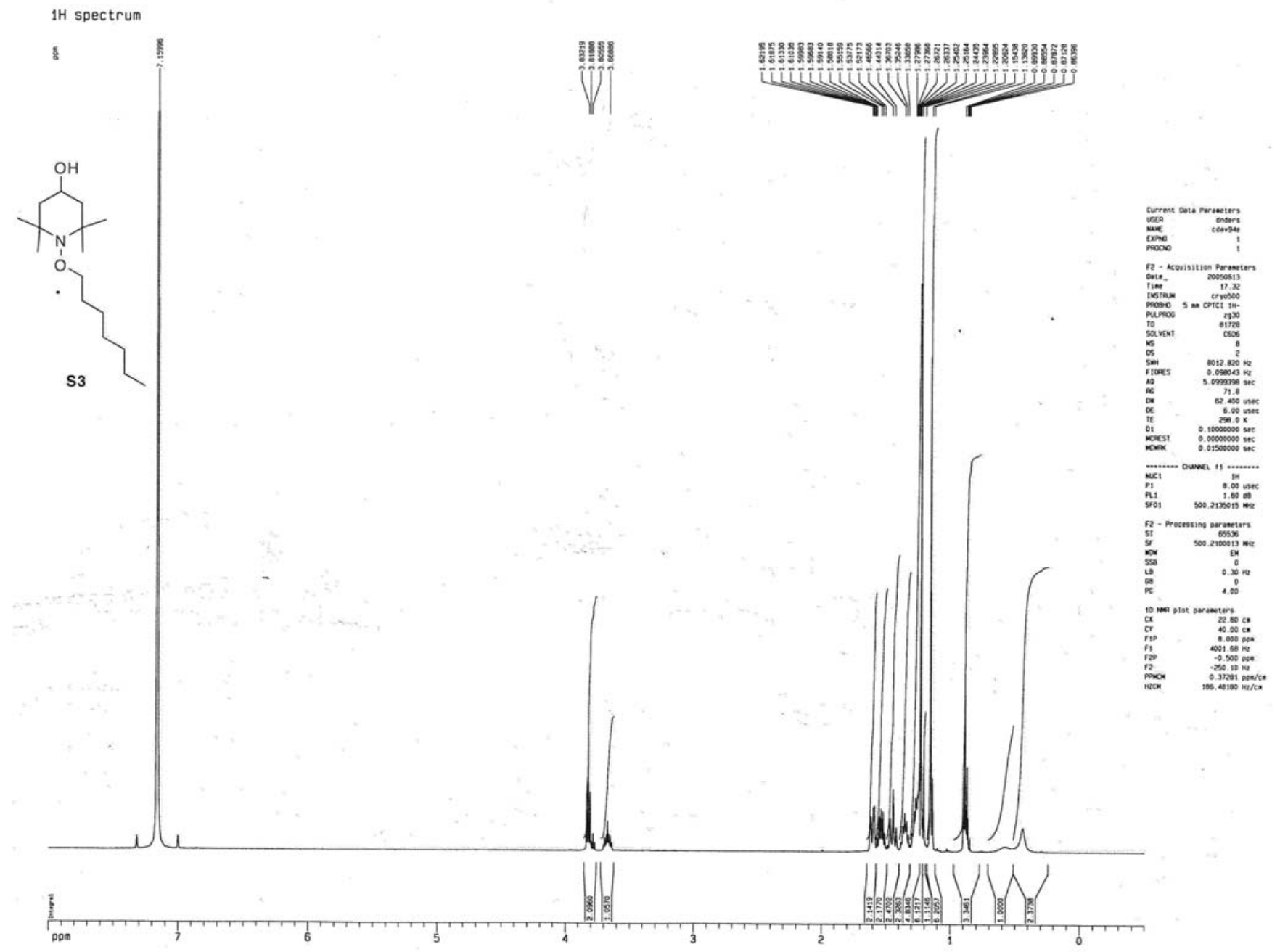




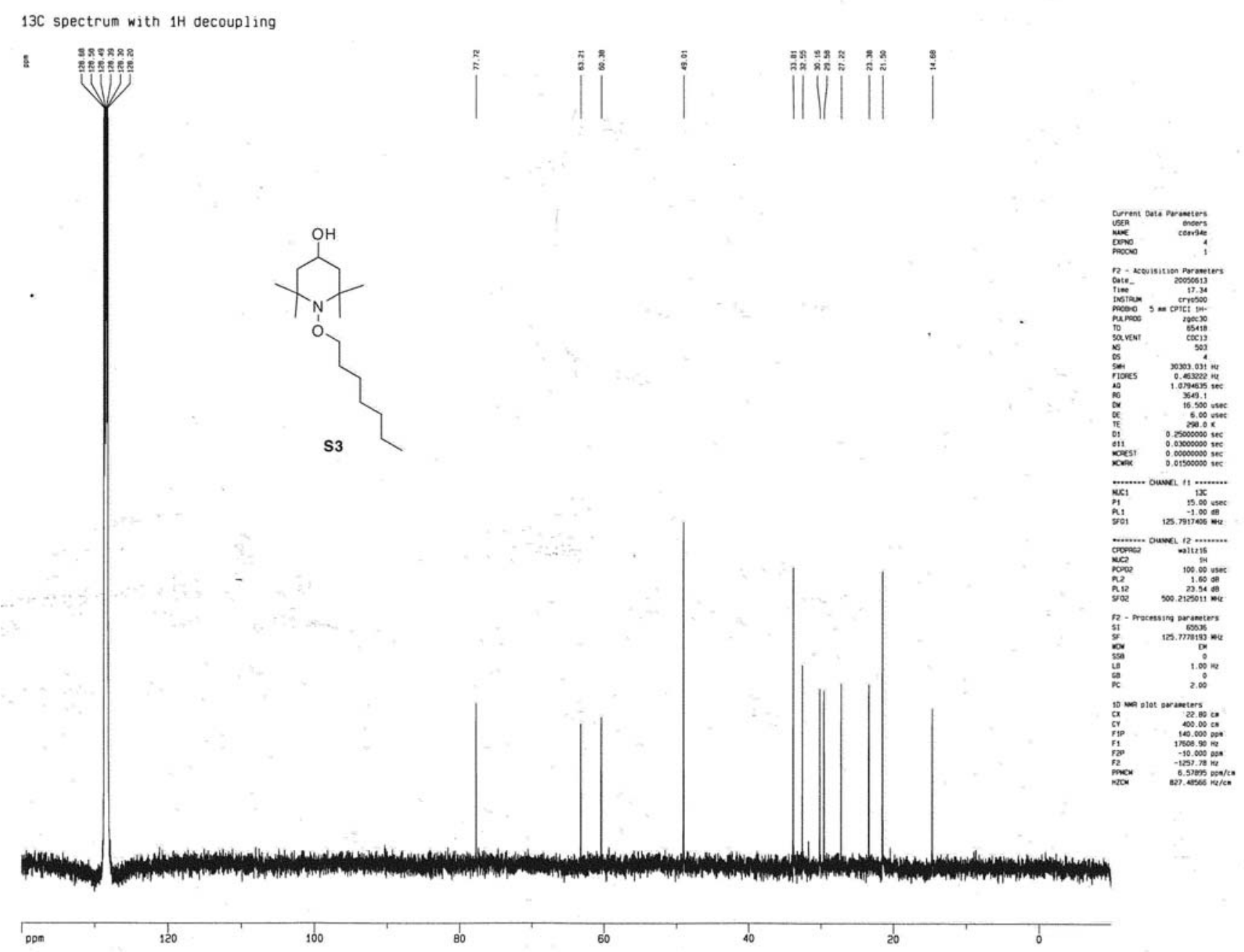


1H spectrum

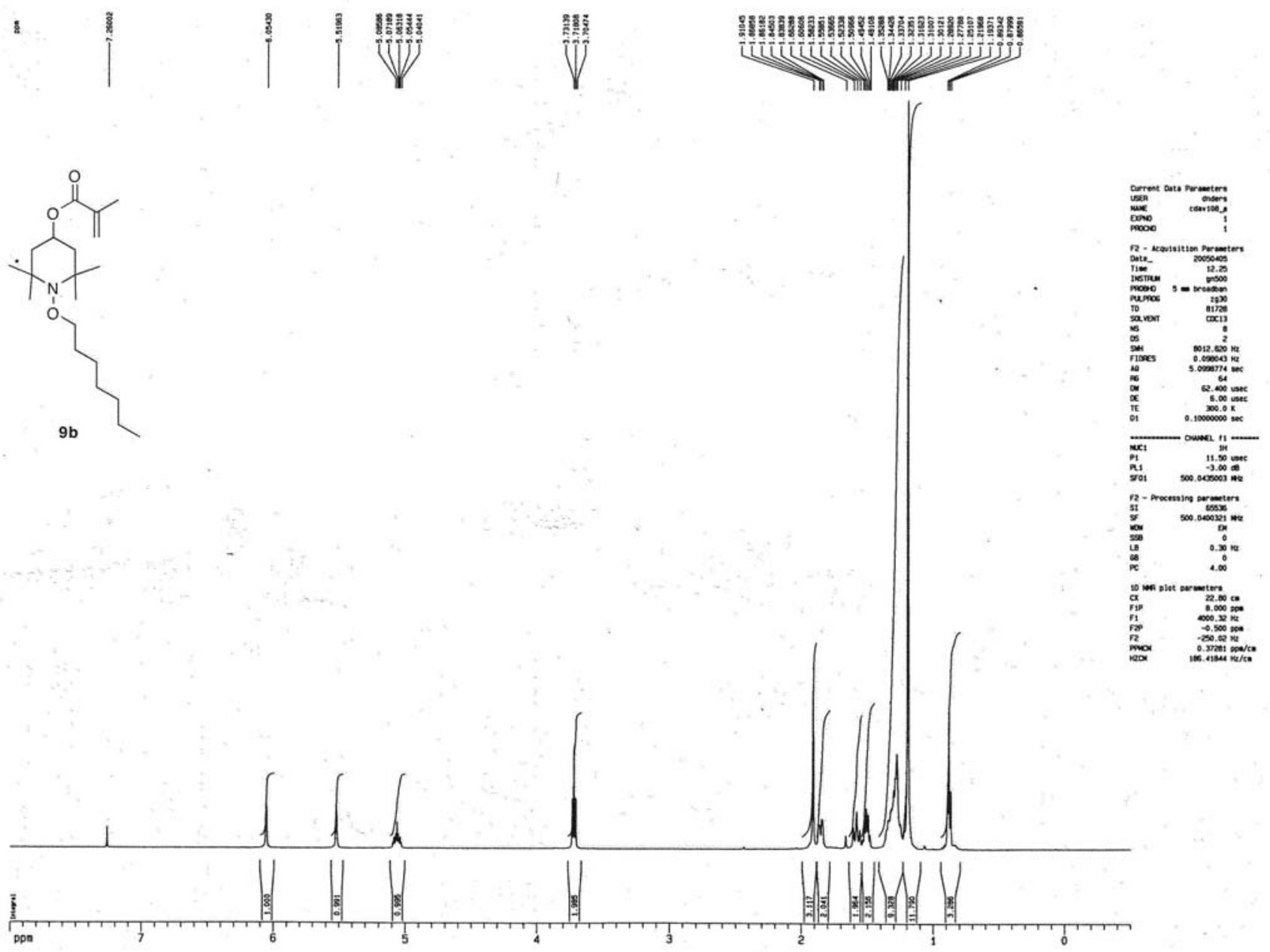


$13 C$ spectrum with $1 H$ decoupling

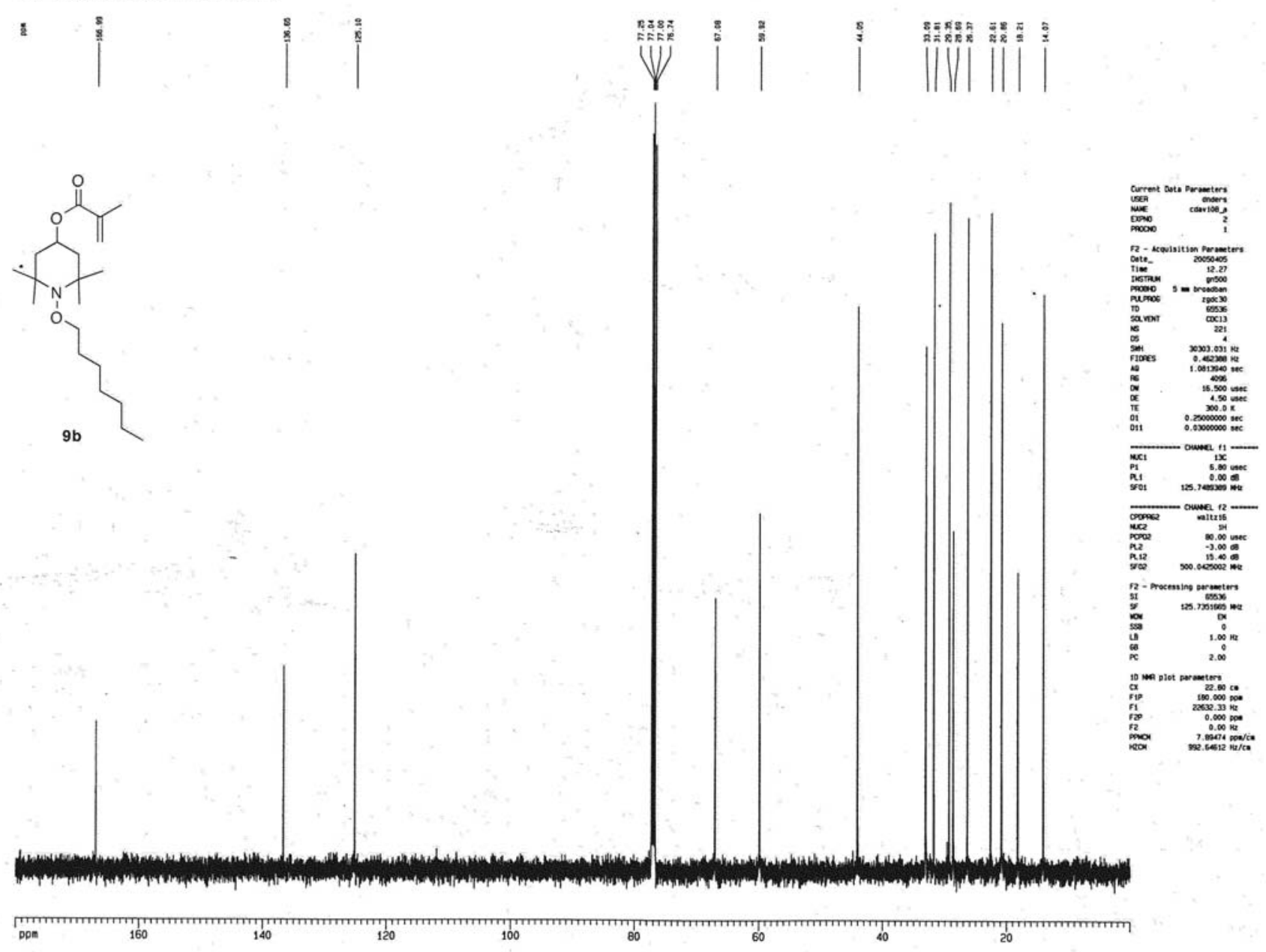


1H spectrum
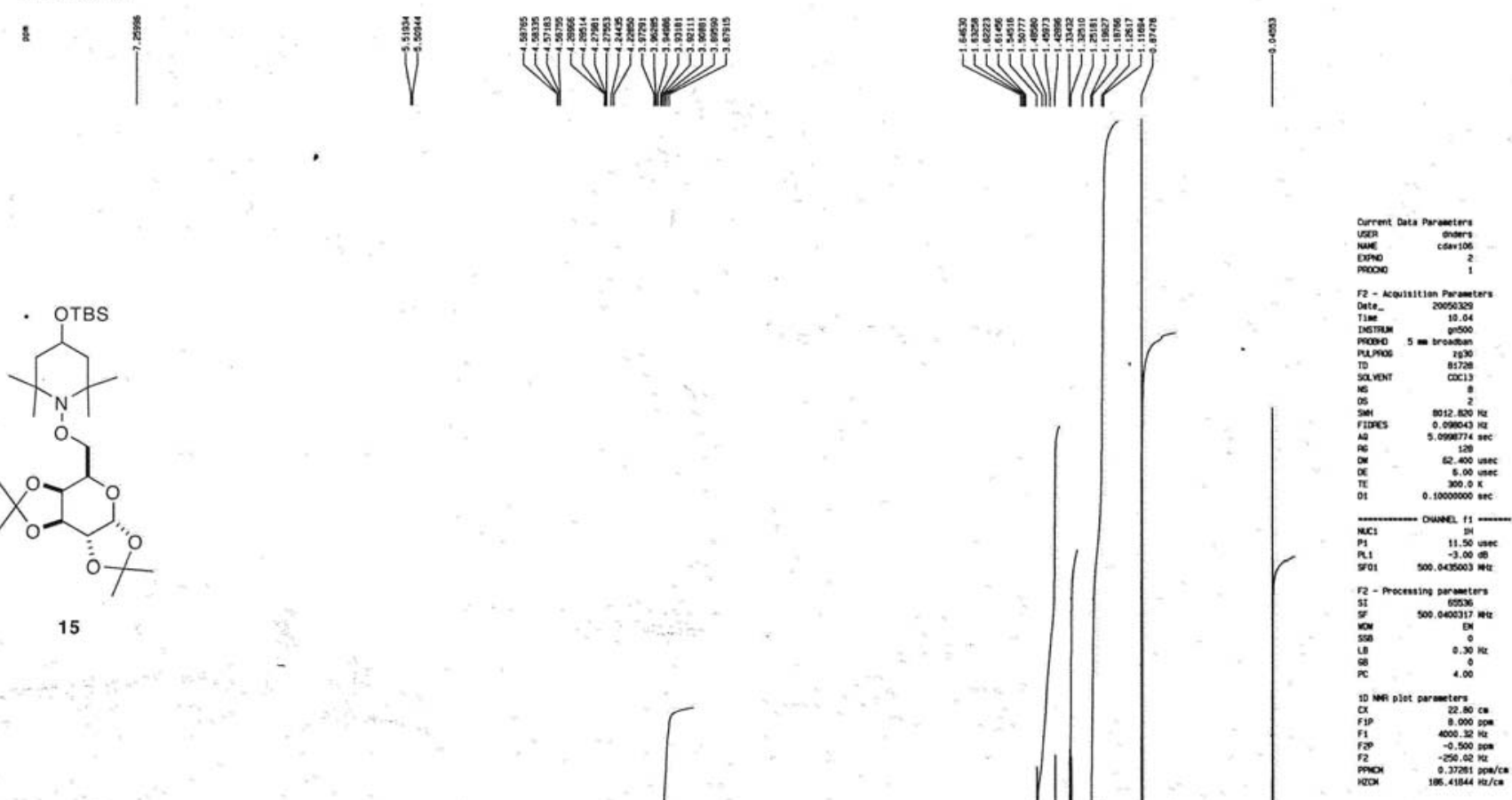

15

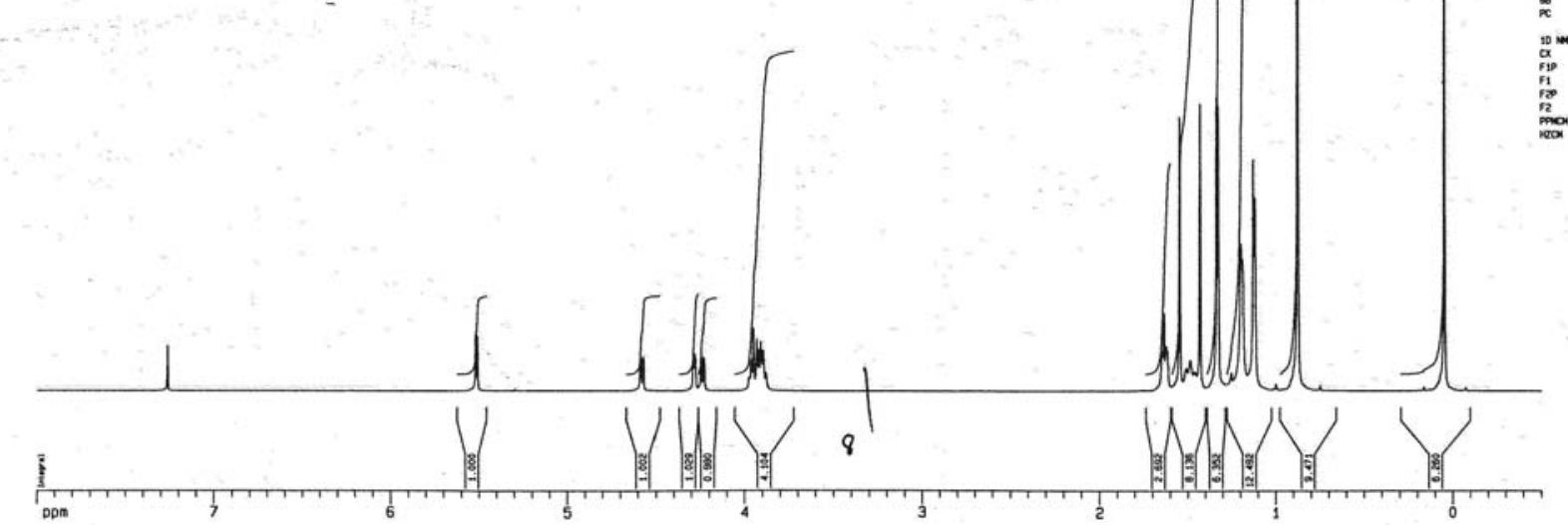


13C spectrum with $1 H$ decoupling
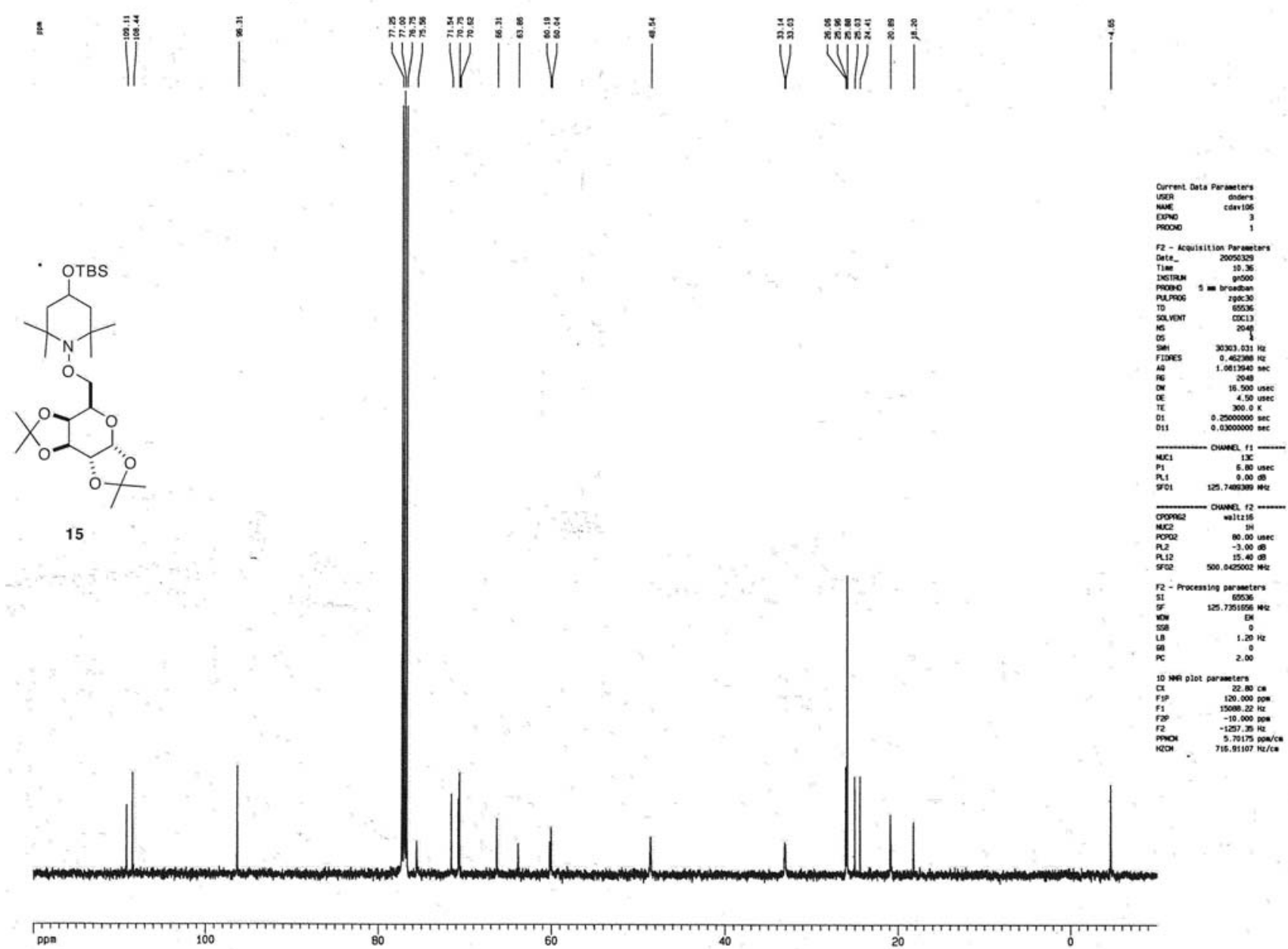


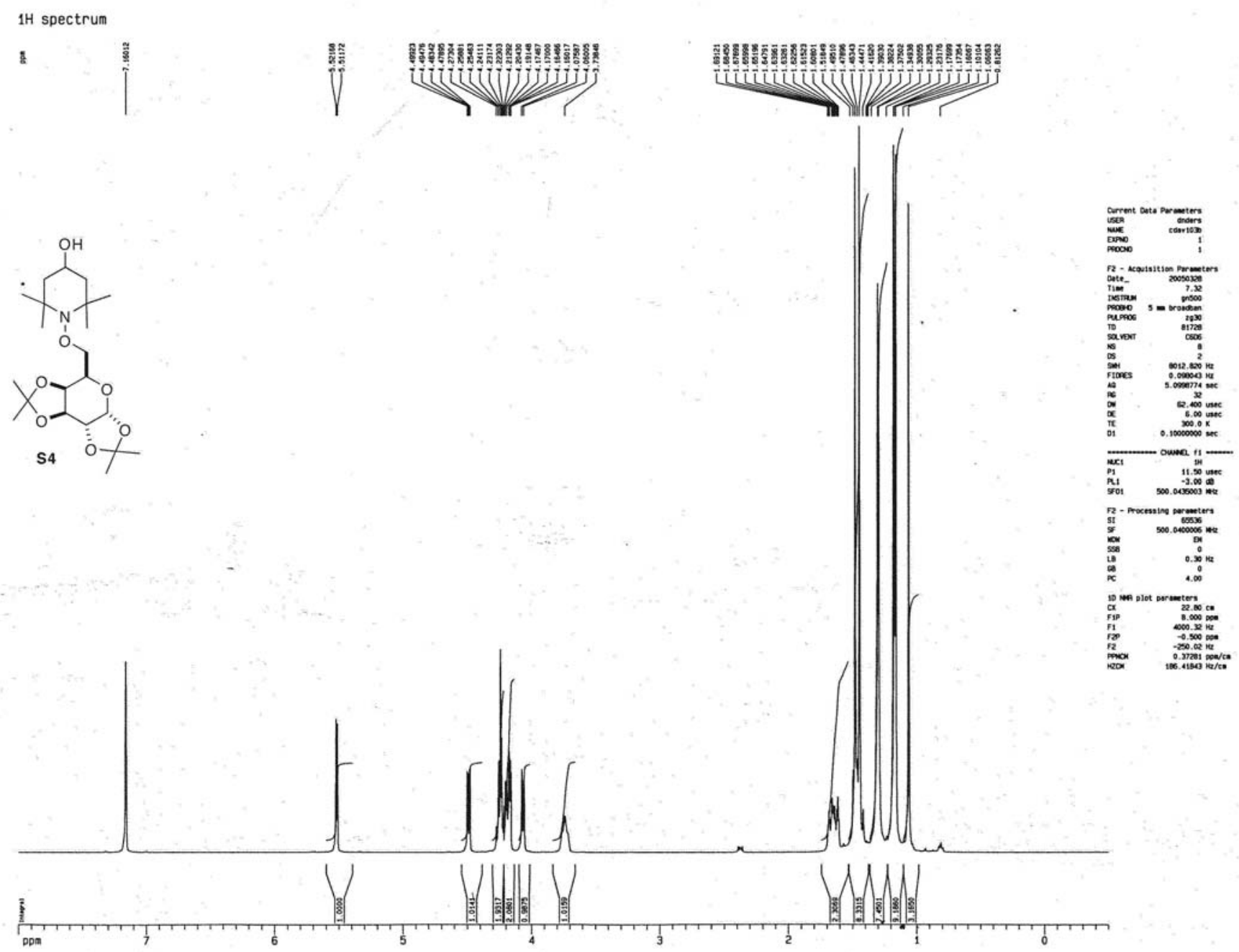




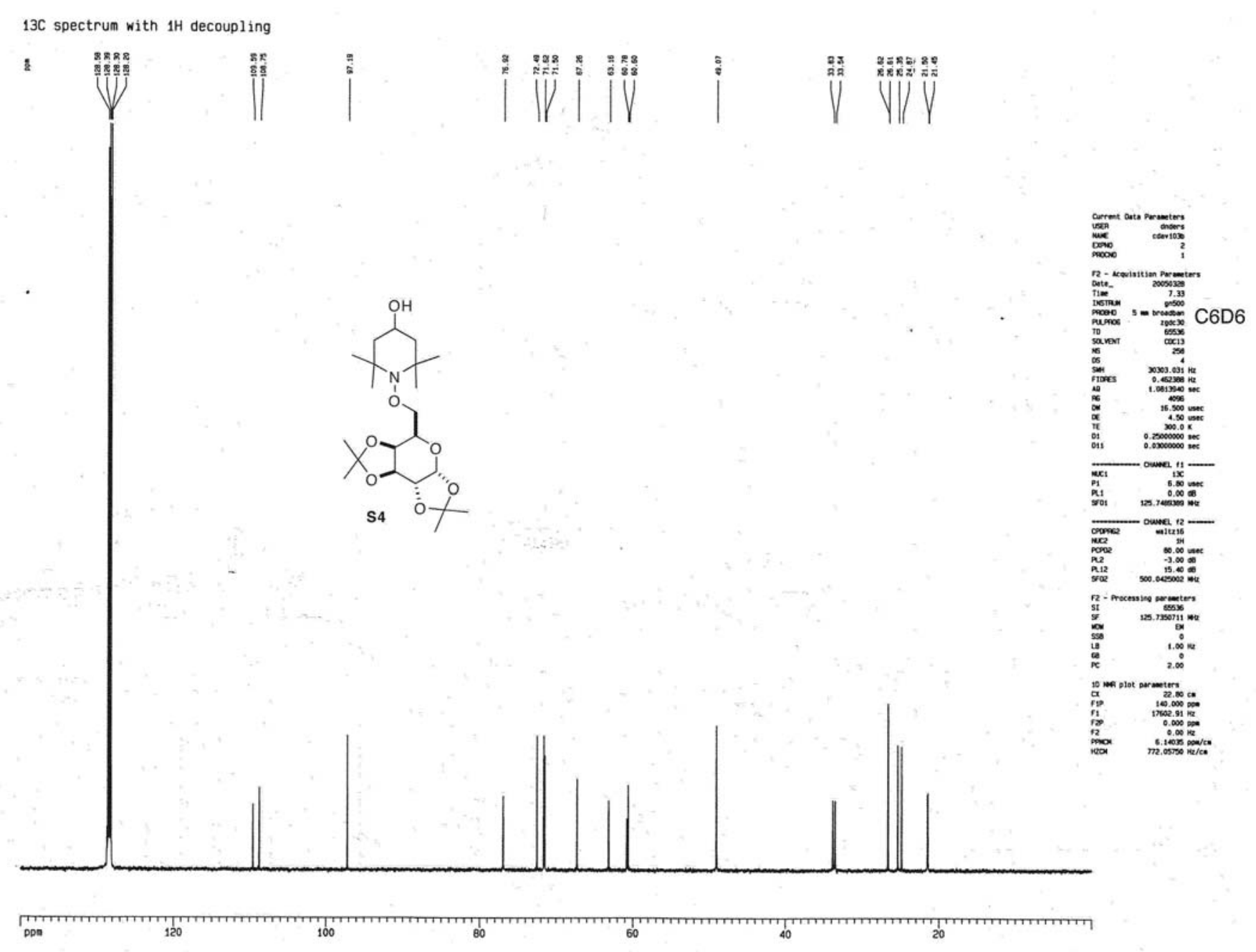




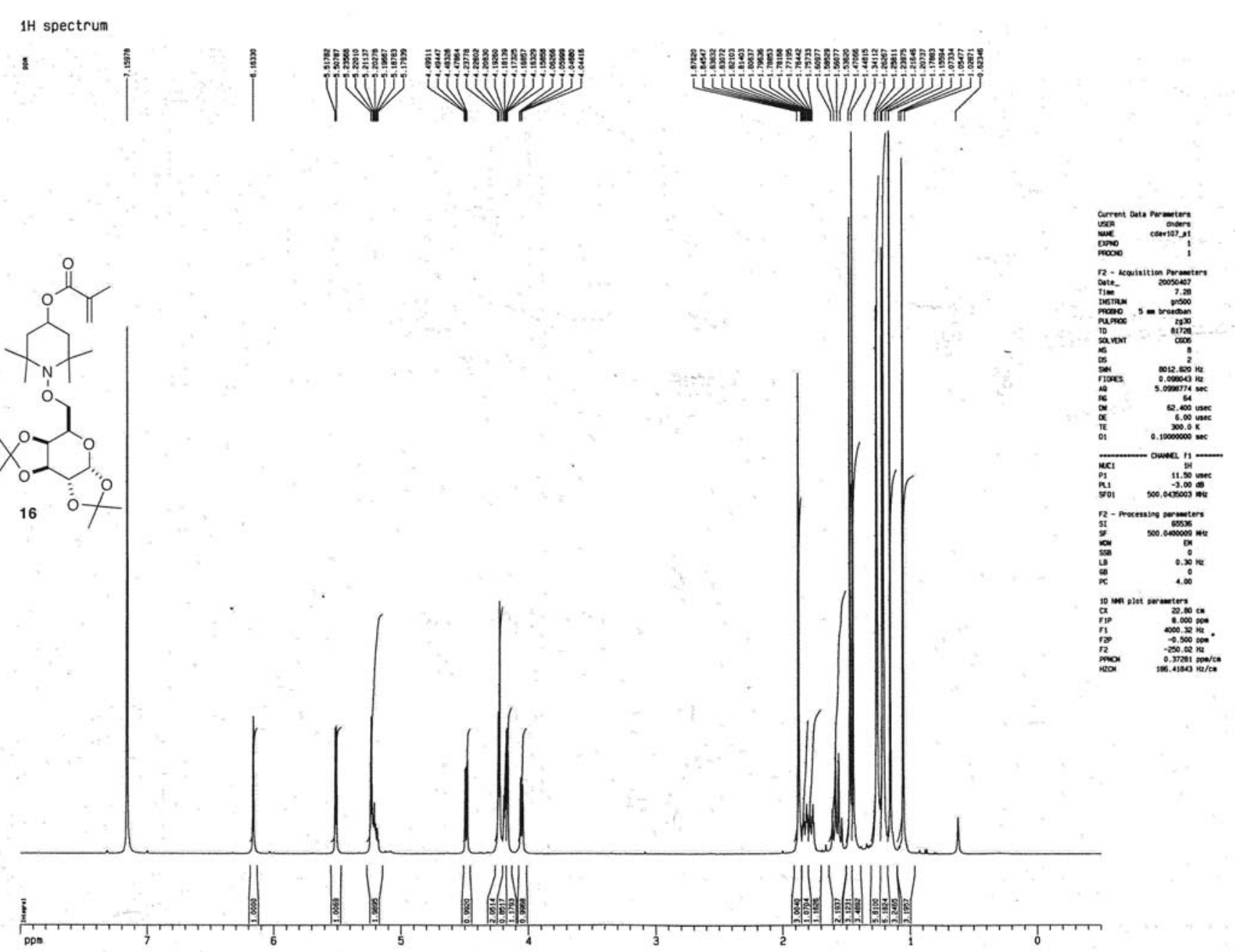




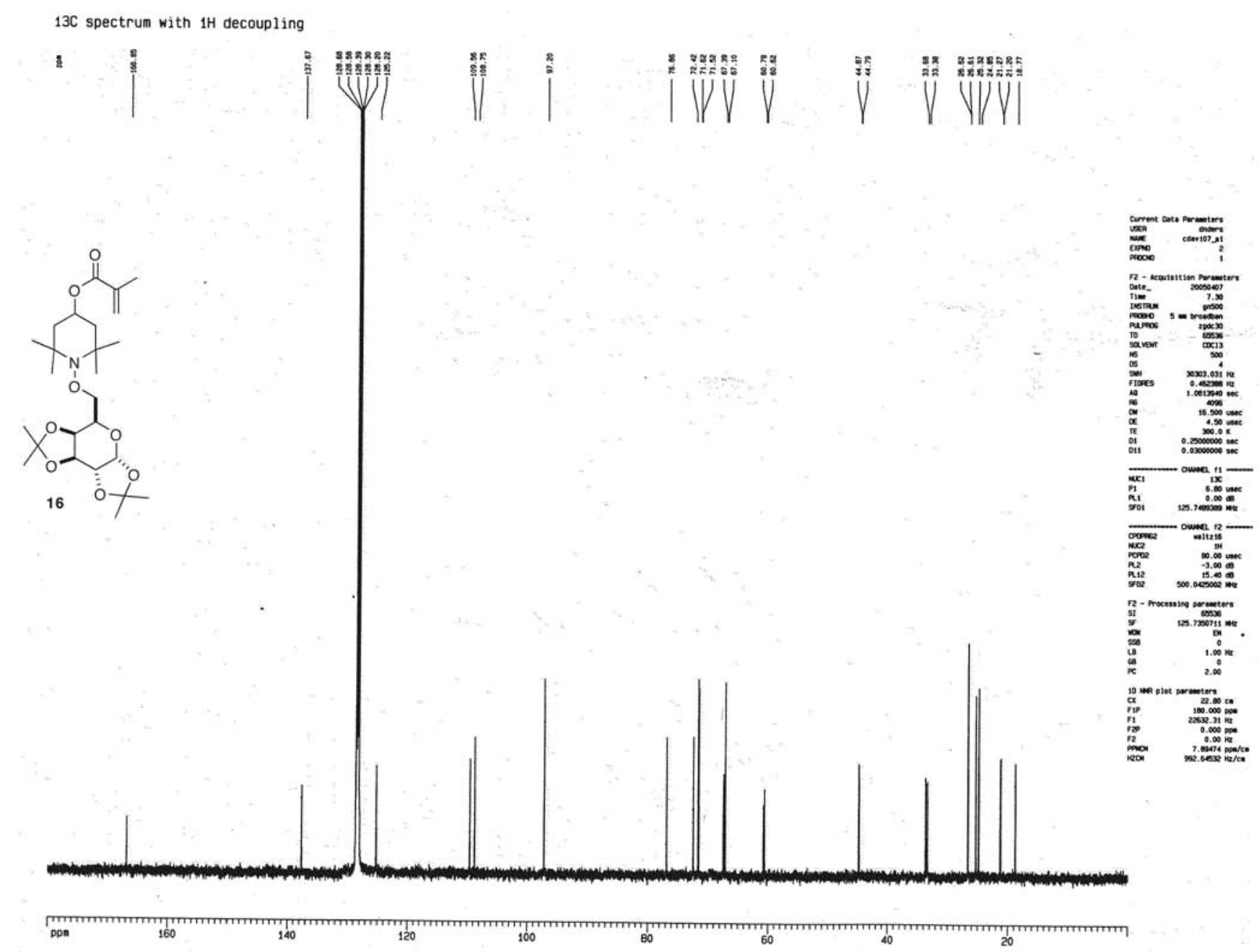


1H spectrum

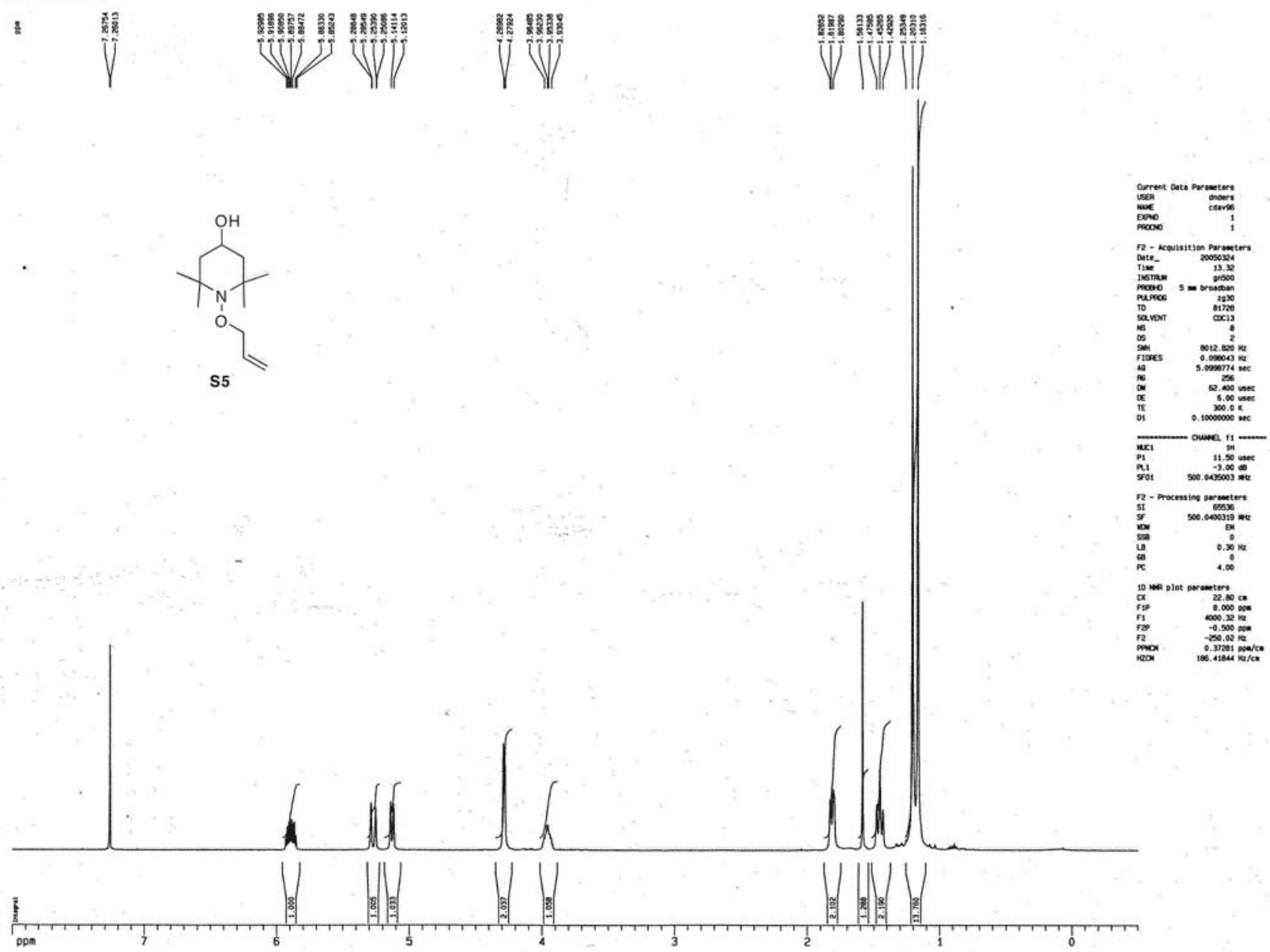


$13 C$ spectrum with $1 H$ decoupling

\&
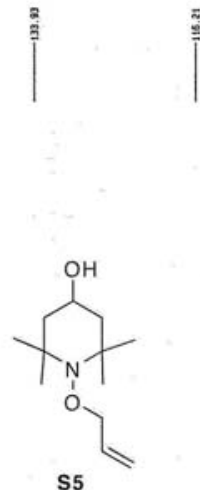

s5

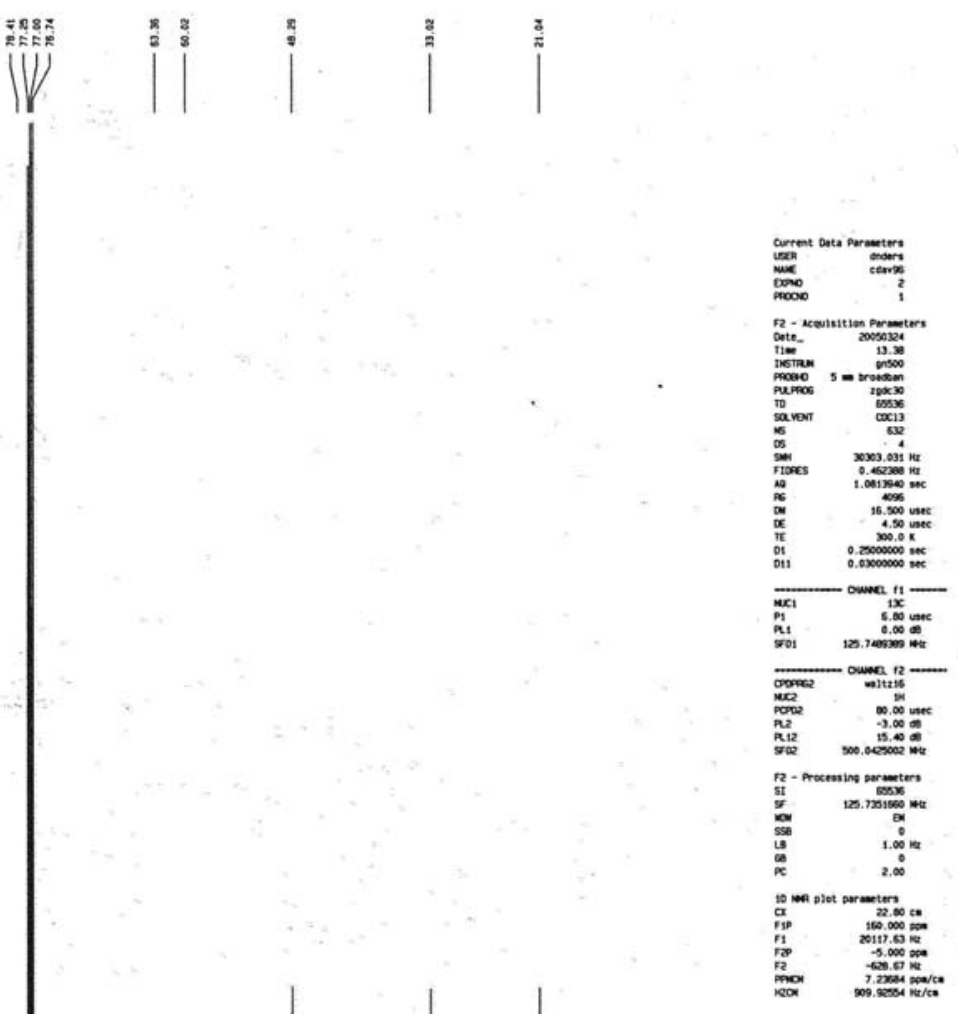


$1 \mathrm{H}$ spectrum

$\stackrel{\frac{1}{4}}{\tilde{1}}$

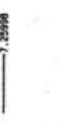

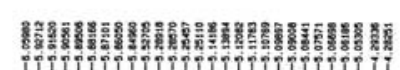

wiviving

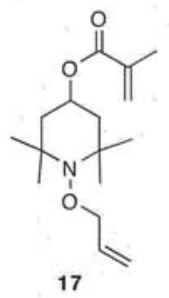

17
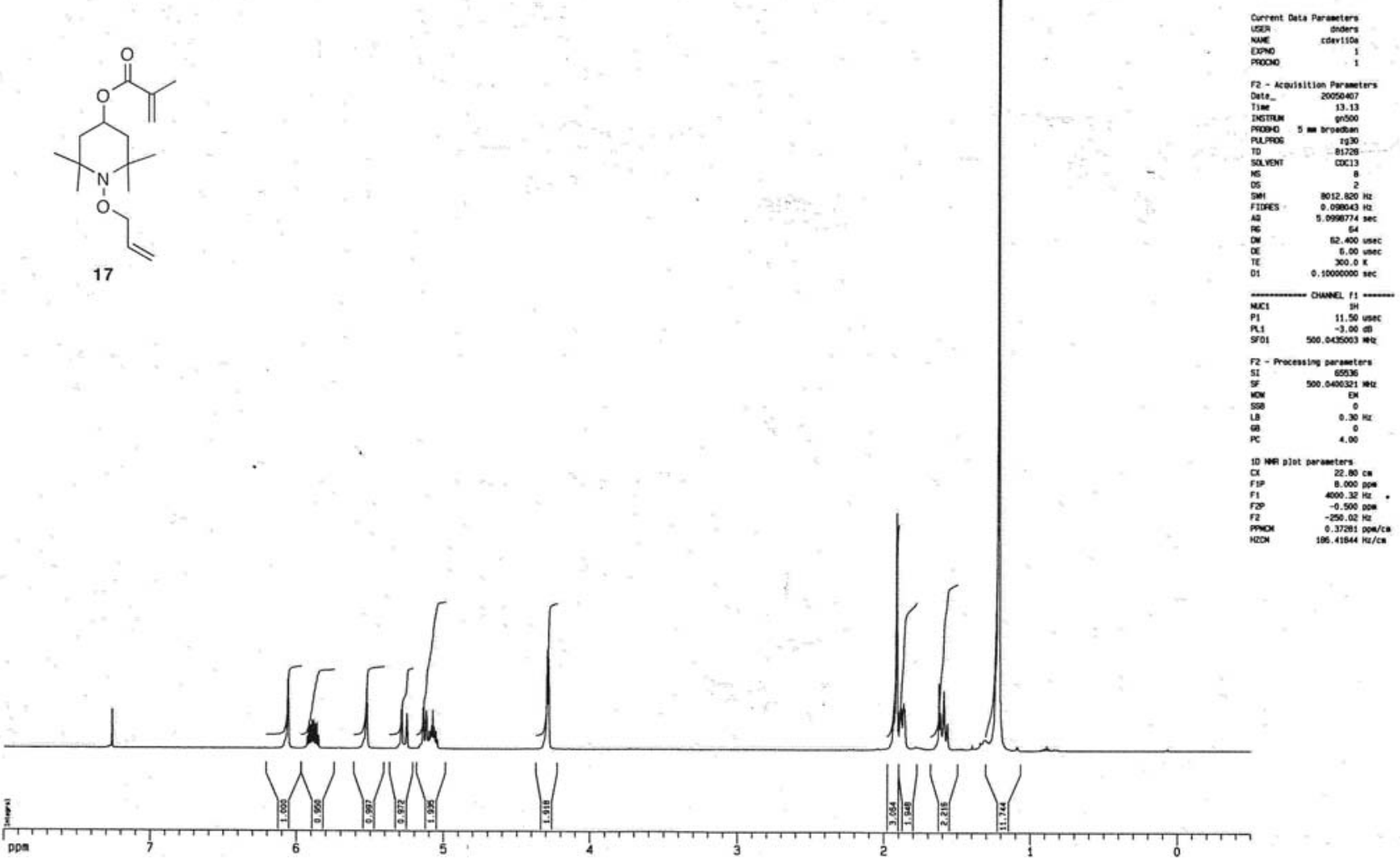
$13 \mathrm{C}$ spectrum with $1 \mathrm{H}$ decoupling

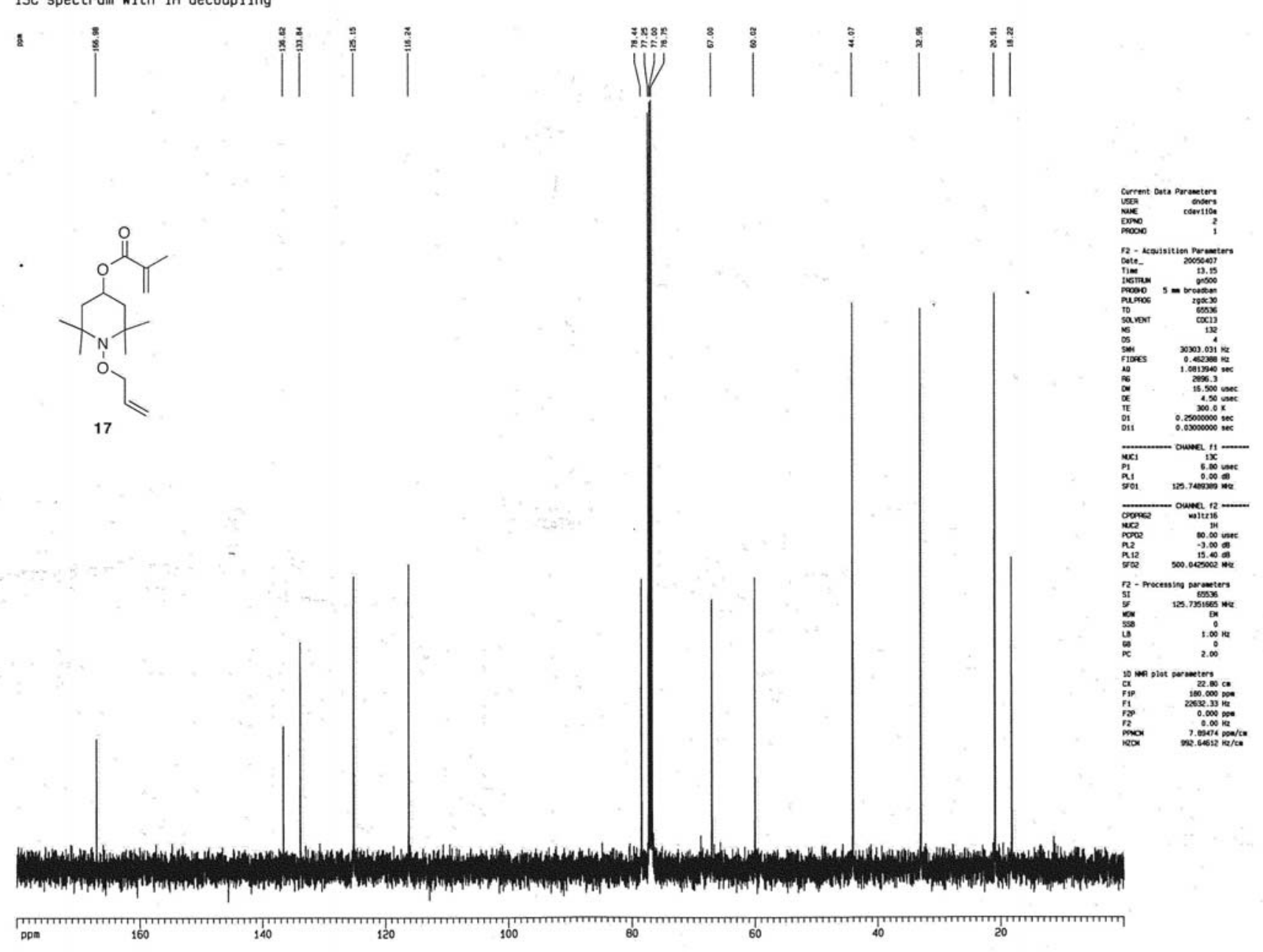




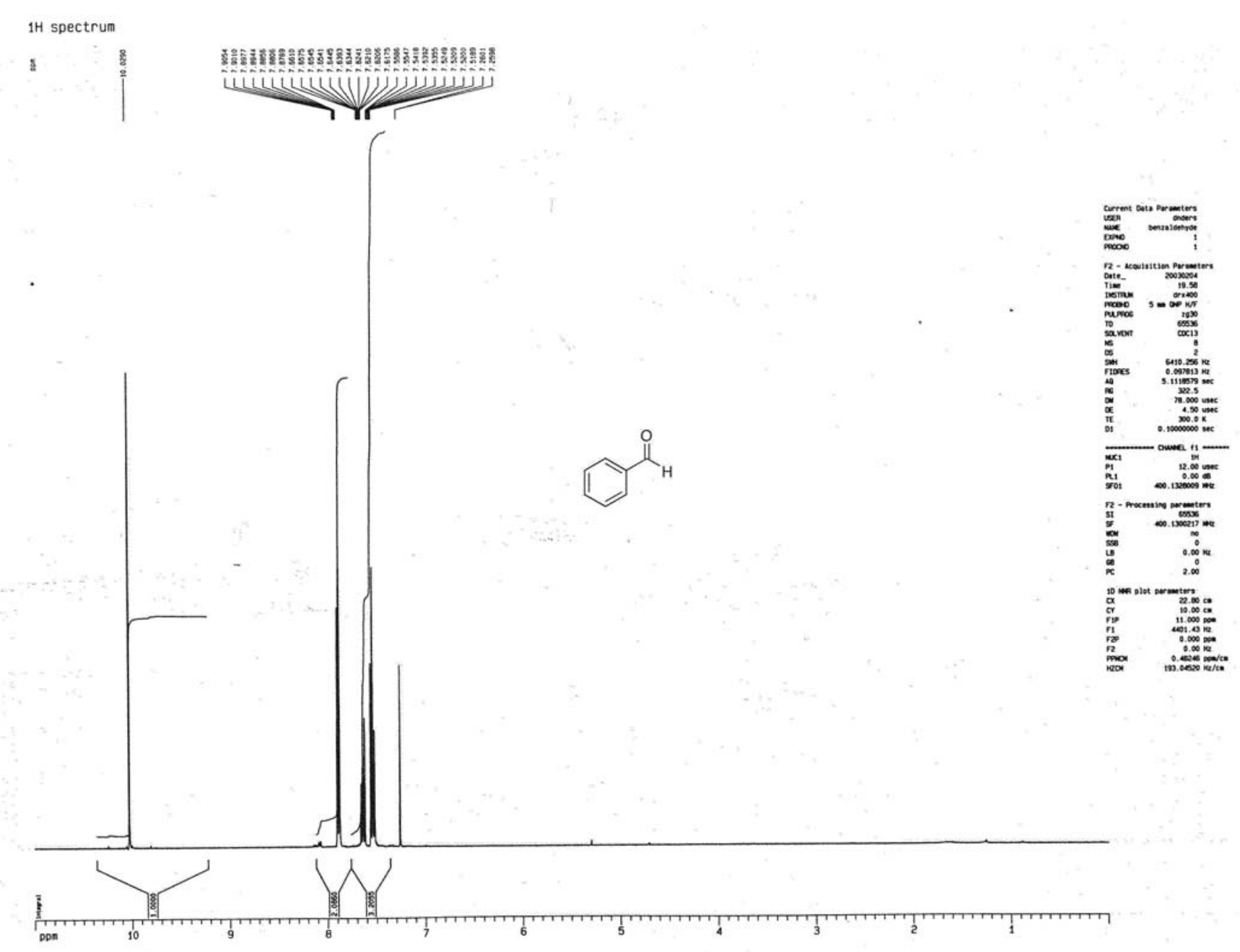




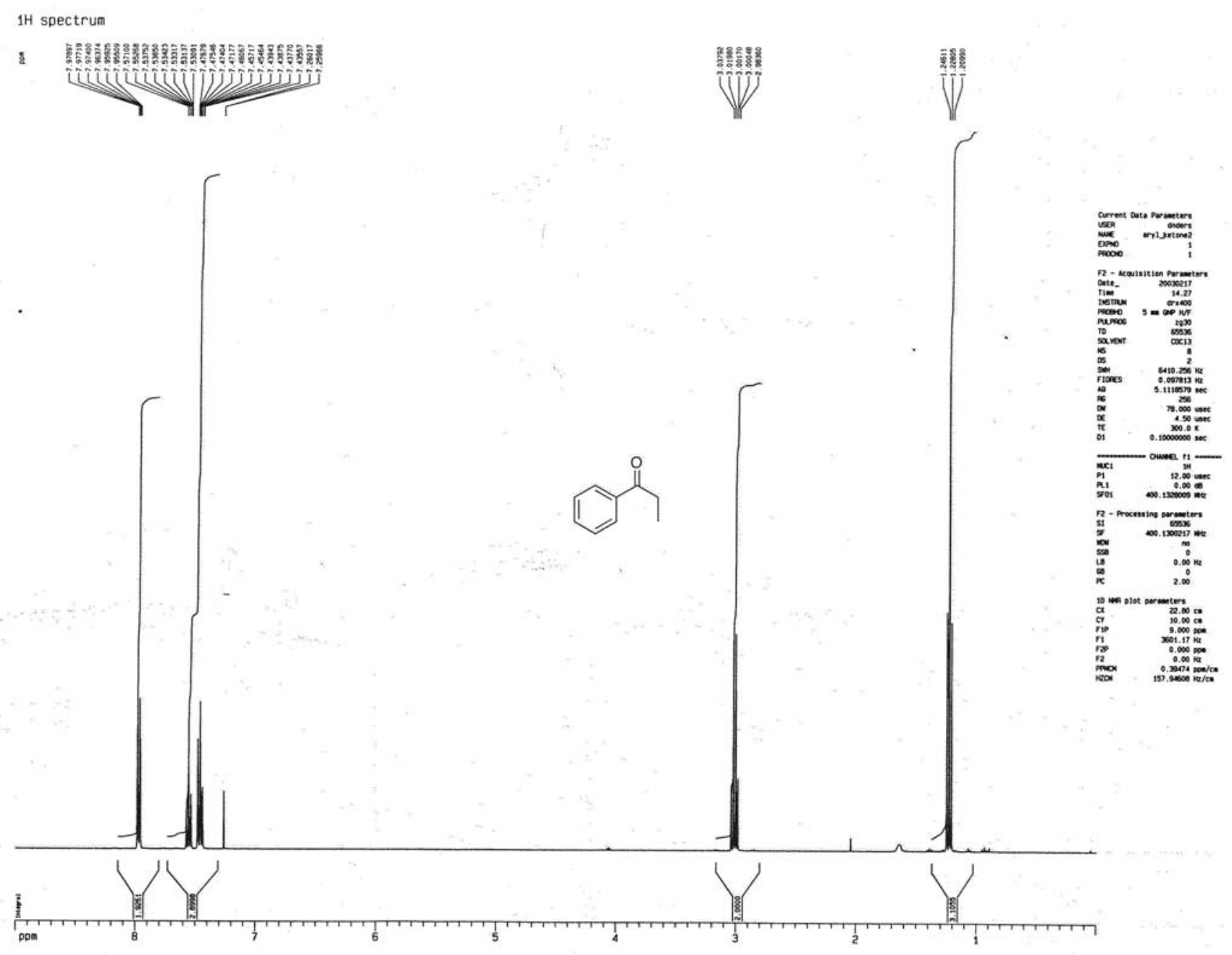


$1 \mathrm{H}$ spectrum

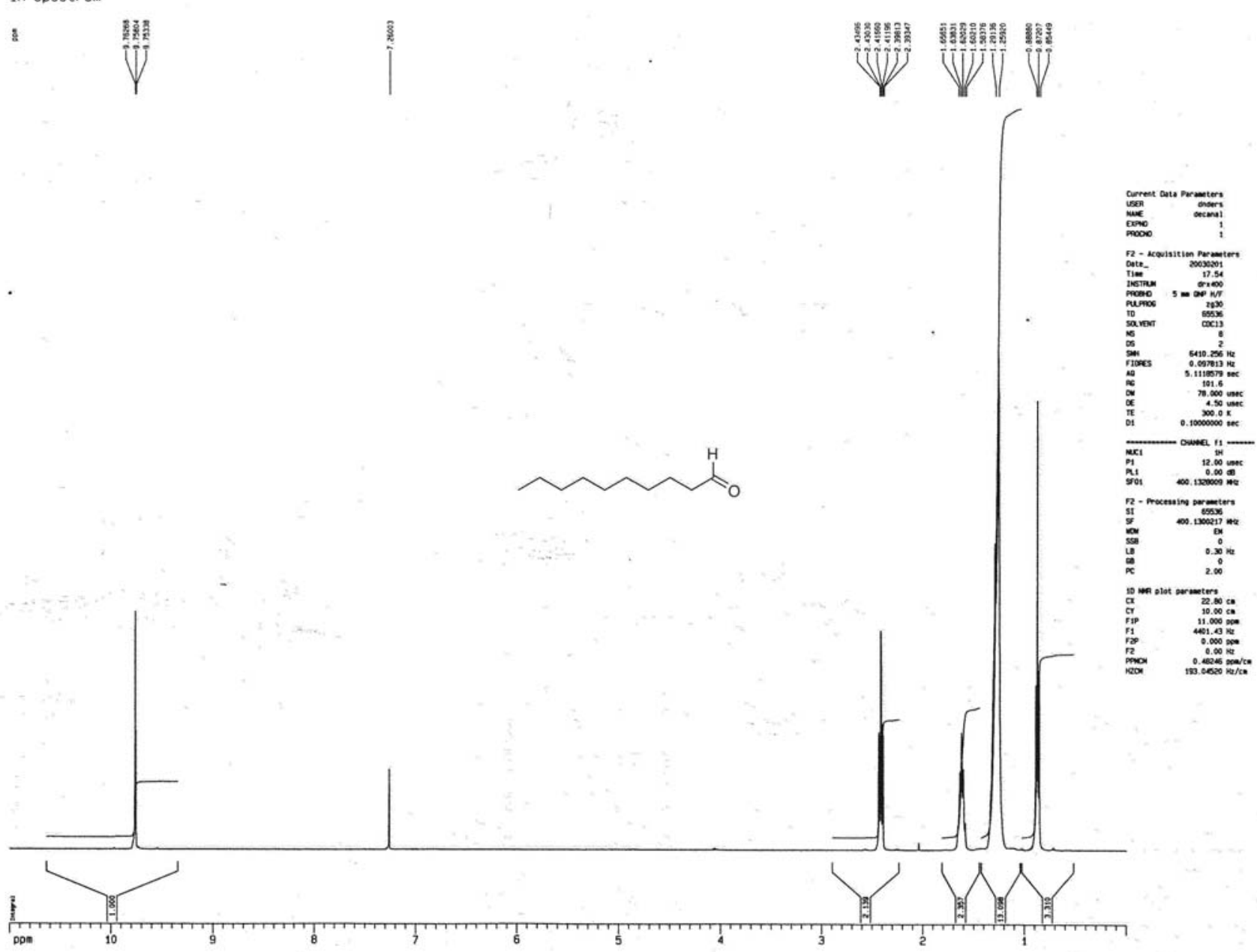




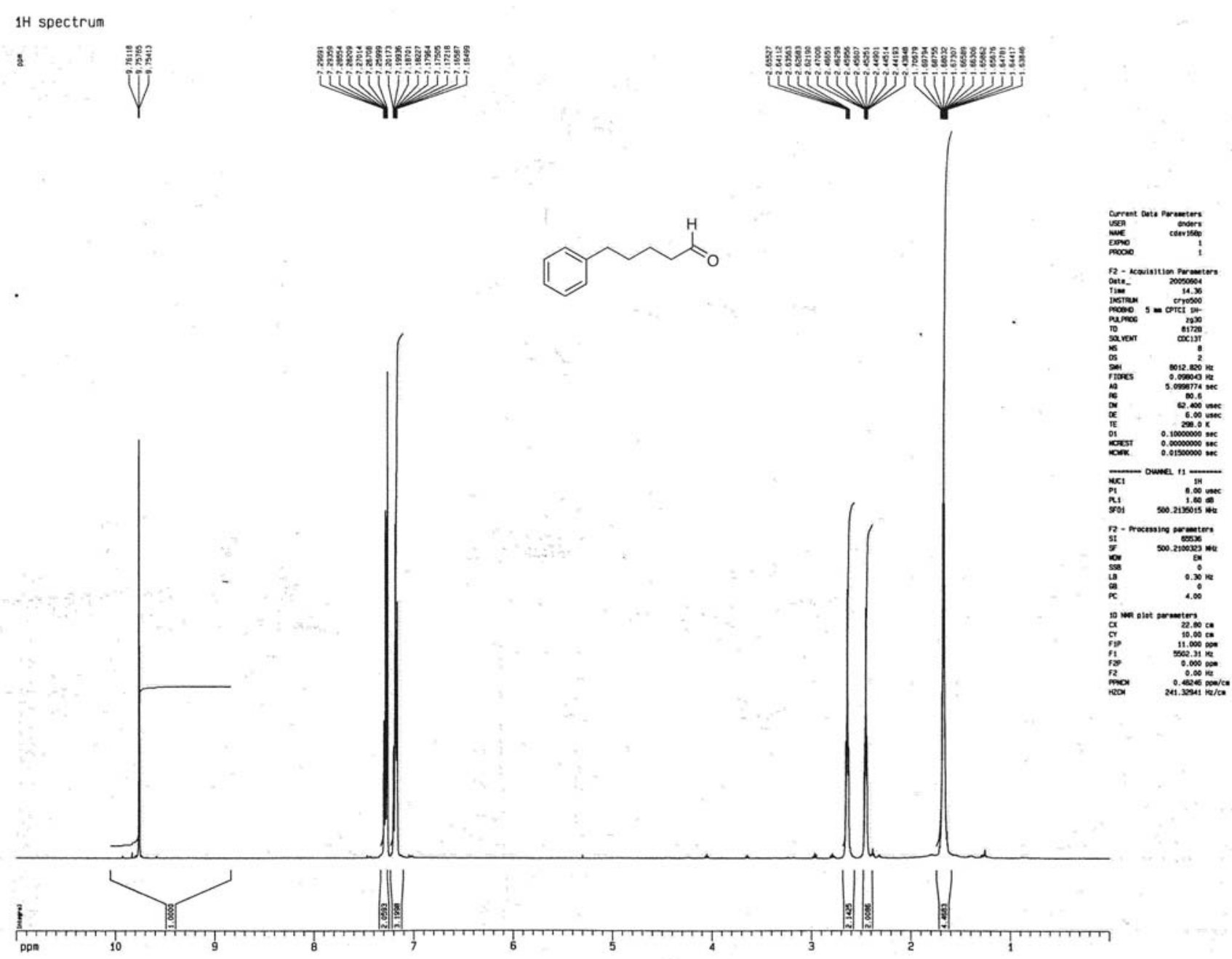




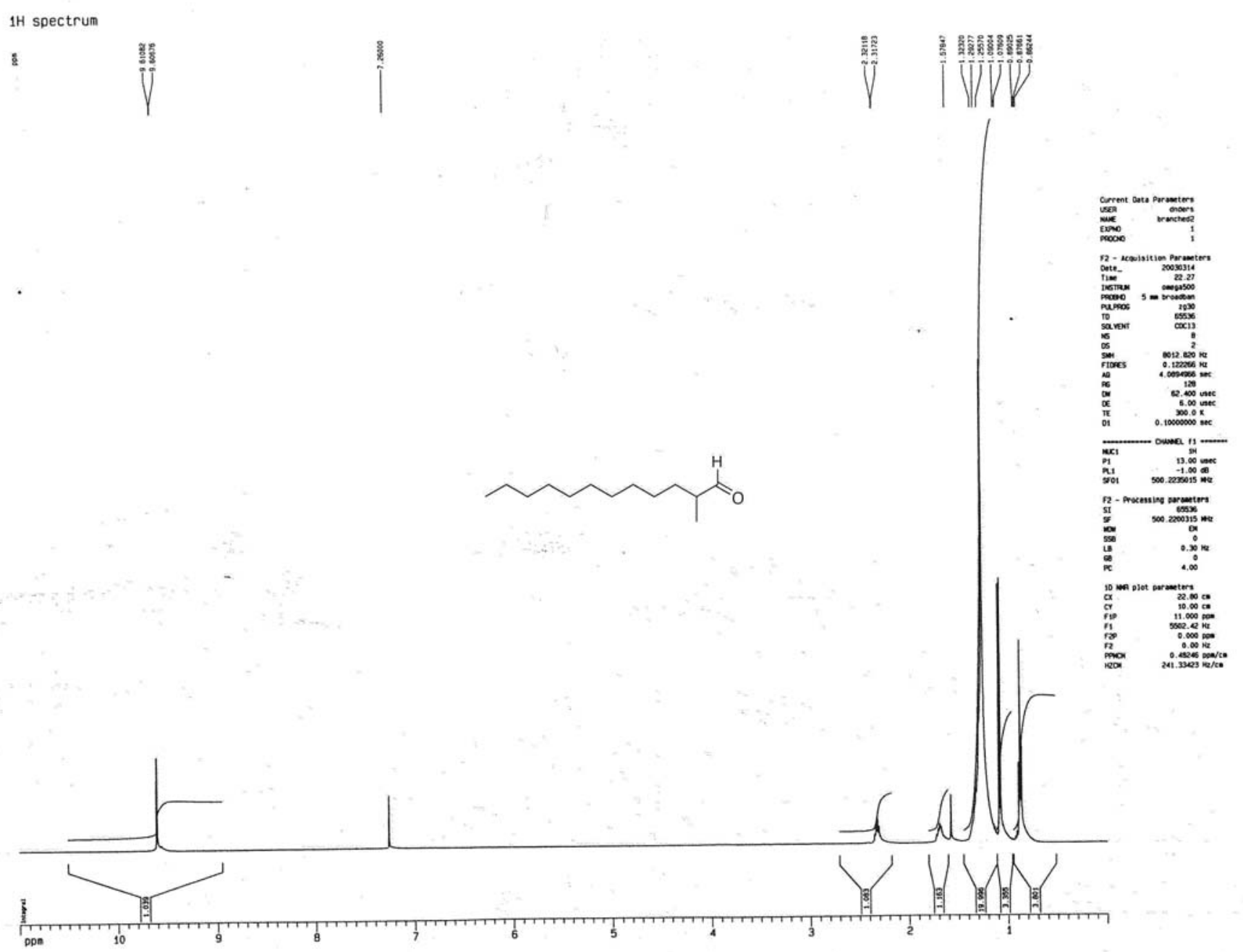

